FERMILAB-TM- 2640-DI

November 2016

\title{
Fermilab Testbeam Facility Annual Report - FY 2016
}

M. G. Albrow ${ }^{1}$, M. Alfred ${ }^{2}$, A. Apresyan ${ }^{1}$, Z. Arcara ${ }^{3}$, B. Azmoun 4 , V. Baily ${ }^{5}$, R. Belmont ${ }^{6}$, C. Biggs ${ }^{4}$, B. Bilki ${ }^{7}$, J. Blackburn ${ }^{5}$, G. Bolla ${ }^{1}$, S. Boose ${ }^{4}$, G. Brunetti ${ }^{1}$, B. Casey ${ }^{1}$, F. Cavanna ${ }^{8}$, C-Y $\mathrm{Chi}^{9}$, M. Chiu ${ }^{4}$, M. Connors ${ }^{9}$, M. Contalbrigo ${ }^{10}$, D. Denisov ${ }^{1}$, E. Desmond ${ }^{11}$, S. Durgut $^{12}$, A. Dychkant $^{13}$, R. Esha ${ }^{14}$, V. Evdokimov ${ }^{15}$,D. Faia ${ }^{13}$, A. Franz ${ }^{4}$, J. Freeman ${ }^{1}$, A. Gaponenko1, C. Gatto $^{16}$, J. Haggerty ${ }^{4}$, K. Hanagaki ${ }^{17}$, D. Harris ${ }^{1}$, M. Hayturadov ${ }^{12}$, X. He ${ }^{9}$, D. Hedin ${ }^{13}$, T. K. Hemmick $^{11}$, M. Higdon ${ }^{5}$, R. Hooper ${ }^{3}$, J. Huang' ${ }^{4}$, Y. Ikegami18, D. Ingram ${ }^{12}$, K. Kauder ${ }^{19}$, H. Kim²0, A. Kisilev, , E. Kistinev ${ }^{4}$, J. Labounty ${ }^{4}$, M. Lenz ${ }^{4}$, W. Lenz ${ }^{4}$, S. Li ${ }^{5}$, C-J Lin ${ }^{21}$, V.

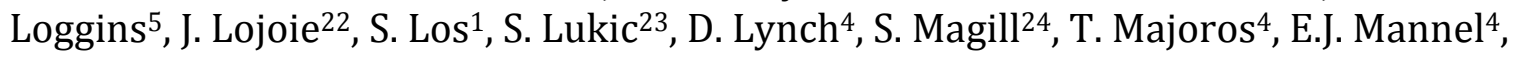

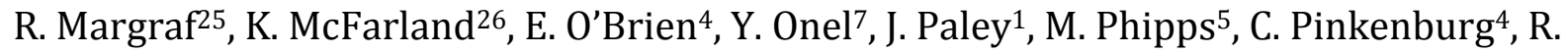
Pisani $^{4}$, S. Polizzo ${ }^{4}$, C. Pontieri, ${ }^{4}$ E. Prebys ${ }^{1}$, J. Price ${ }^{27}$, M. Purschke ${ }^{4}$, J.L. Raaf ${ }^{1}$, E. Ramberg ${ }^{1}$, J. Repond $^{24}$, T. Rinn ${ }^{22}$, R. Rivera ${ }^{1}$, A. Ronzhin 1 , R. Ruggiero 4 , V. Samoykenko ${ }^{28}$, M. Sarsour ${ }^{9}$ A. Sen $^{22}$, A. Sickless ${ }^{5}$, M. Skoby ${ }^{29}$, C. Snyder ${ }^{12}$, P. Sobel ${ }^{5}$, M. Spiropulu ${ }^{30}$, S. Stoll ${ }^{4}$, A. Sukhanov ${ }^{1}$, S. Syed ${ }^{9}$, E. Thorsland ${ }^{5}$, F. Toldo ${ }^{21}$, O. Tsai ${ }^{14}$, P. Ujic ${ }^{23}$, B. Ujvari ${ }^{4}$, L. Uplegger ${ }^{1}$, S. Uzunyan ${ }^{13}$, S. Carson-Vazquez ${ }^{4}$, J. Waters ${ }^{3}$, L. Wen ${ }^{14}$, C. P. Wong ${ }^{9}$, C. Woody ${ }^{4}$, J. Xie ${ }^{24}$, S. Xie ${ }^{30}$, K. Yaiima ${ }^{31}$, A. Zhang 32

Editors: S. Geer ${ }^{1}$, M. Rominsky ${ }^{1}$, C. Tate ${ }^{1}$

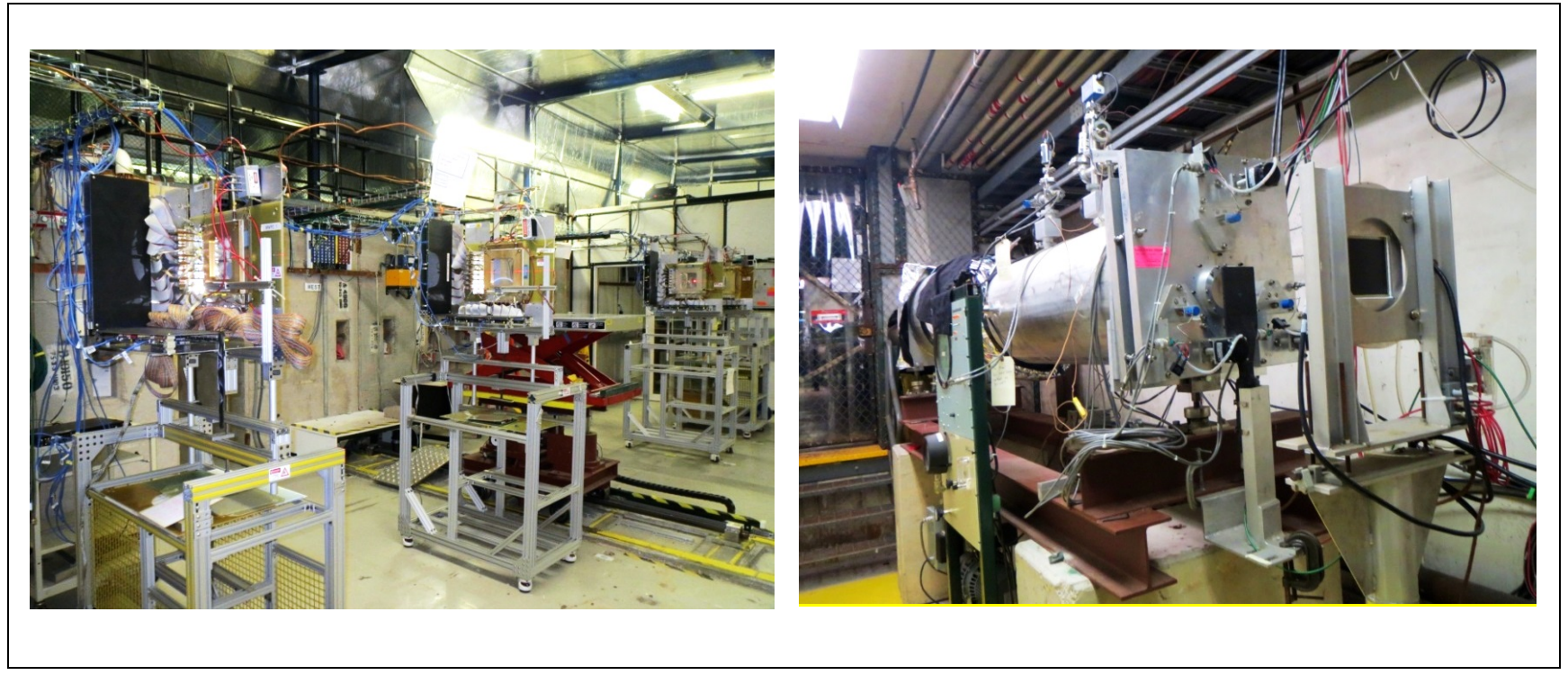

${ }^{1}$ Fermilab, ${ }^{2}$ Howard University, ${ }^{3}$ Lewis University, ${ }^{4} \mathrm{BNL}, 5$ University of IL, 6 University of CO/Boulder, ${ }^{7}$ University of IA/IA City, ${ }^{8}$ FNAL/INFN, ${ }^{9}$ GA State University, ${ }^{10}$ INFN/Ferrara, ${ }^{11}$ Stoney Brook University, ${ }^{12}$ University of IA, ${ }^{13} \mathrm{~N}$. Illinois University, ${ }^{14} \mathrm{UCLA}$, ${ }^{15} \mathrm{IHEP} /$ Protvino, ${ }^{16} \mathrm{INFN} /$ Lecce, ${ }^{17} \mathrm{KEK} /$ Osaka University, ${ }^{18} \mathrm{KEK},{ }^{19} \mathrm{~W}$ ayne State University, 20 University of Chicago, ${ }^{21}$ LBNL, ${ }^{22}$ IA State University, ${ }^{23}$ University of Belgrade, ${ }^{24} \mathrm{ANL} / \mathrm{HEP}$, ${ }^{25}$ Lehigh/FNAL, ${ }^{26}$ University of Rochester, ${ }^{27}$ University of Liverpool, ${ }^{28}$ IHEP/Moscow, ${ }^{29}$ University of Michigan, ${ }^{30}$ Caltech, ${ }^{31}$ Osaka University, ${ }^{32} \mathrm{FL}$ Institute of Technology 


\section{INTRODUCTION}

This Technical Memorandum (TM) summarizes the Fermilab Test Beam operations for FY 2016. It is one of a series of annual publications intended to gather information in one place. In this case, the information concerns the individual experiments that ran at FTBF and are listed in Table TB-1. Each experiment section was prepared by the relevant authors and was edited for inclusion in this summary.

\subsection{The Fermilab Test Beam Facility in Fiscal Year 2016}

The Fermilab Test Beam facility continues to provide users with a place to characterize, calibrate, and develop new detector technologies. In FY16, MTest and MCenter took physics quality data with users publishing 11 articles and working on another 4 . We have also worked to implement a number of items, based on user feedback and recommendations from the committee. The following were tasks accomplished:

- We performed numerous beam studies and are able to produce particle composition plots for many of the beam energy values.

- A new system was put into place to request resources. This will ultimately lead to less paperwork and an easier time for users to get the resources they need. This new online system will speed up safety walkthroughs and provide a clear record of the findings.

- We optimized the performance of the Cherenkov detectors.

- An integrated DAQ for the facility was implemented and used during the last few days before the shutdown.

\subsection{Beam Characteristics at the Test Beam}

The MTest beamline runs in three different beam modes: the primary beam $(120 \mathrm{GeV}$ protons), the pion mode (8-66 GeV mixed particle beam), and the low energy pion mode (1$32 \mathrm{GeV}$ mixed particle beam).

Reviewing the last two years of test beam users shows that the primary beam of $120 \mathrm{GeV}$ protons and the low energy pion beam are the most requested beam energies, as shown in Figure TB-1. 


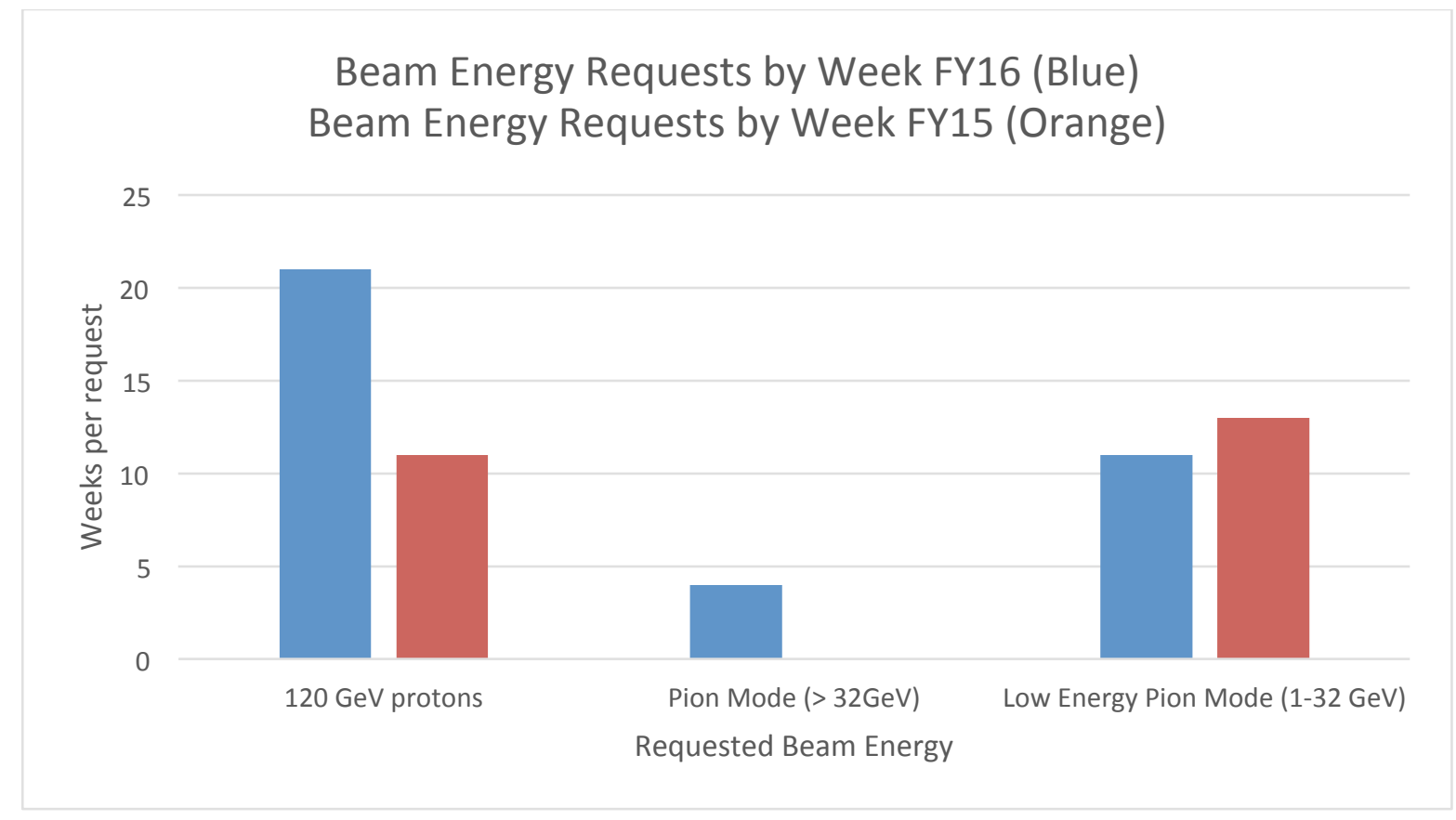

Figure TB-1: The beam energy requests for FY15 and FY16 per week. Note that in FY15 we installed and commissioned the MINERvA detector, which resulted in fewer weeks of beam time.

In order to better serve test beam customers, we have launched a series of studies to make sure the beam is well characterized. While the full range of beam energies will be studied over time, our focus has been on the low energy pion mode as our most requested and least understood beam energy. There were a series of studies done by D. Jensen and E. Skup throughout FY16 to determine particle content in those energies. The plots are shown below in Figure TB-2 and Figure TB-3. In addition to the particle content, we would like to understand the momentum spread as a function of requested beam. To that end, we are building a spectrometer that can be installed in section MT6.2D when that area is available.

MT6.2D area was chosen because it is closest to user's setups and easy for FTBF staff to access and maintain. Magnets or detectors in the spectrometer can be easily repaired or changed out in this area. This would not be the case if the spectrometer was located upstream in the restricted and crowded beamline area. Spectrometer magnets are being found and we will initially use wire chambers as the detector. The focus of the spectrometer will be to understand beams of $1-12 \mathrm{GeV}$.

In addition to the spectrometer, we will pursue a Time of Flight System that will focus on low energy particle identification. Our goal is to complete these two new instruments before January 2017. 
When beam returns at the beginning of November, we will use the three weeks before users start to complete more beam studies. One of our users has requested a study of electron purity for beams up to $66 \mathrm{GeV}$. We will work on this study first using the lead glass calorimeter and a muon tagger. Our focus will be on the low energy regime (less than 10 $\mathrm{GeV}$ ) once we implement the Time of Flight system. Please see Section Three for our long term plans.

\section{Positive Beams Composition, Open Collimators 2016}

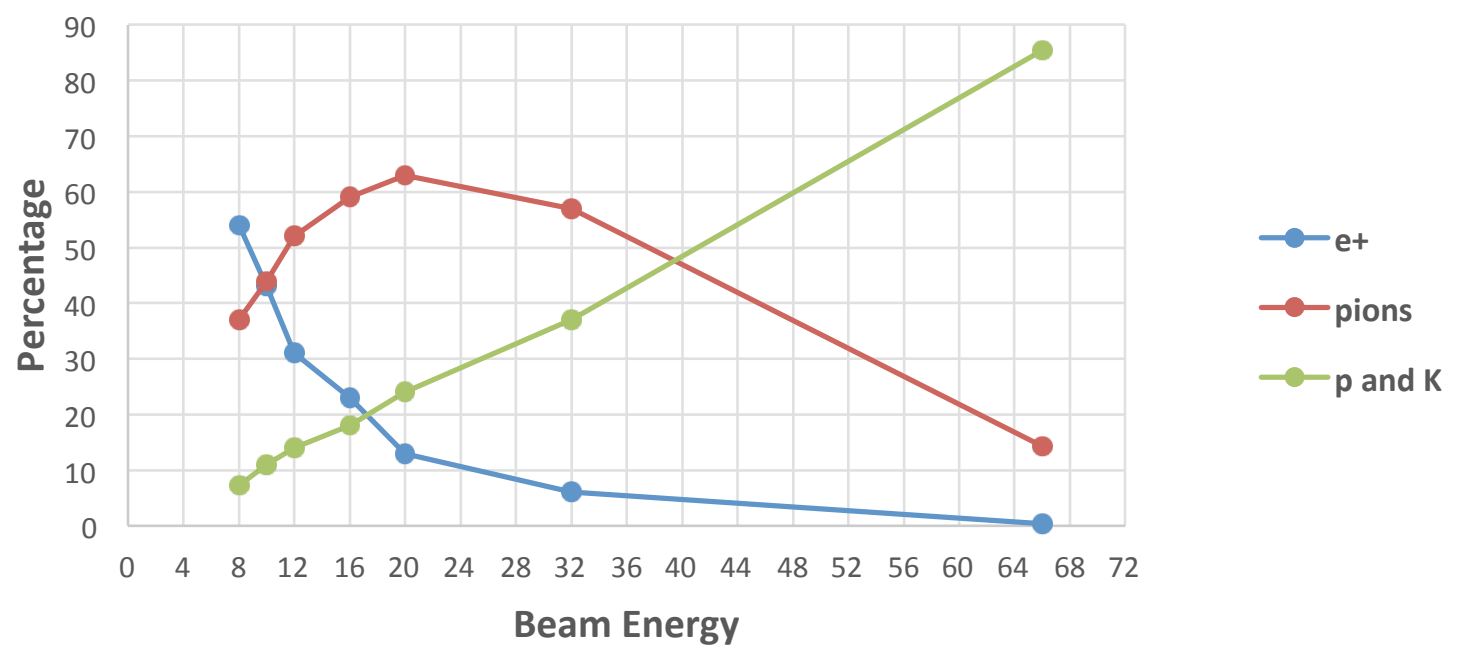

Figure TB-2: Positive beam composition with the collimators open. Data taken with both the upstream and downstream Cherenkov detectors. Studies and analysis done by D. Jensen and E. Skup. 


\section{Negative Beams Composition, Open Collimators}

\section{6}

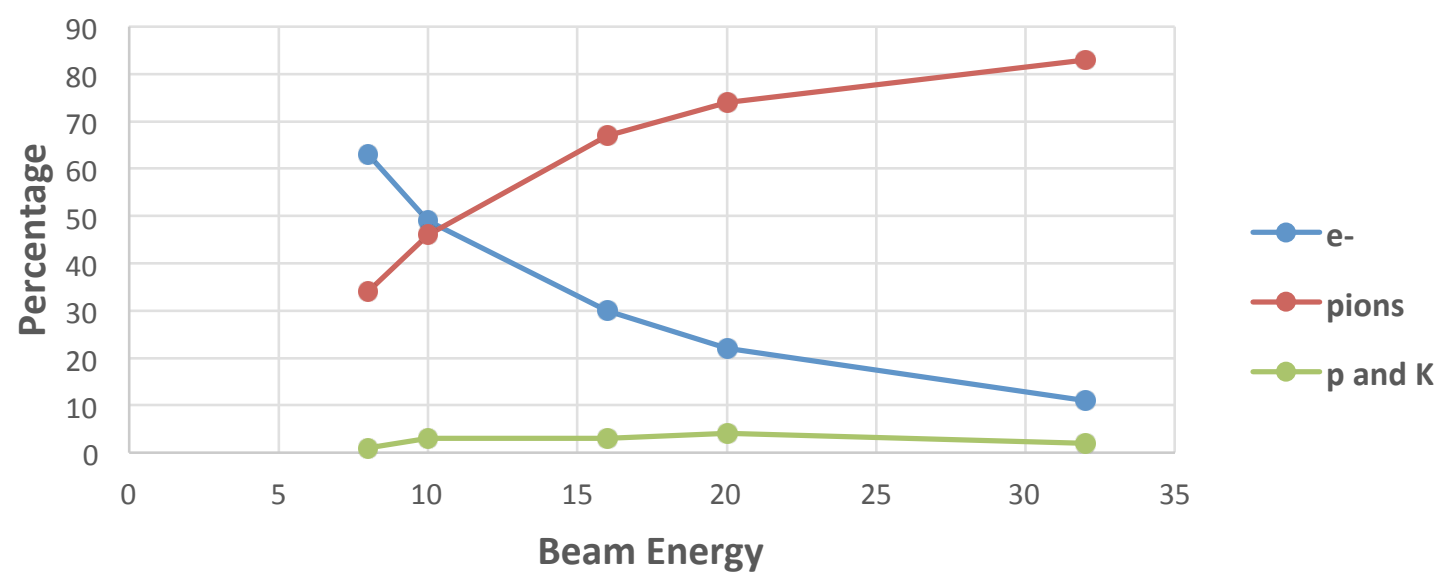

Figure TB-3: Beam composition for the negative polarity beam with the collimators open. Studies and analysis done by D. Jensen and E. Skup.

\subsection{Research Performed at the FTBF in FY 2016}

Each test beam experiment is required to prepare a Technical Scope of Work (TSW) with the Laboratory in which the beam, infrastructure, and safety requirements are spelled out in detail. Four new experiments were approved in FY2016 and starting taking data. Twelve experiments returned to continue their programs. These 16 experiments are listed in Table TB- 4 and represent 261 collaborators from 32 institutions in 6 countries. Figures TB- 6 and TB-7 show the experiments by user classification and research focus. We supported groups from both ATLAS and CMS, as well as groups from Brookhaven and groups looking at future colliders. The Mu2e experiment tested a variety of detectors and is continuing to use the facility to develop their data acquisition system. The g-2 experiment used the facility to conduct rate of rise tests for their straw tube detectors. We fully supported the neutrino experiments LArIAT and LAToF for ProtoDUNE. We also had several groups come to test general detector research and development. Full information is included in the second half of this report. 


\begin{tabular}{|c|c|}
\hline Experiment Number & Description \\
\hline T1041 & CMS Forward Calorimetry R\&D \\
\hline T0992 & Radiation Hard Sensors for the HL-LHC \\
\hline T1015 & Dual Readout Calorimetry with Glasses \\
\hline T1065 & Secondary Emitters Study \\
\hline T1068 & Beam Tests of the SVX4 Telescope \\
\hline T1043 & Mu2e Cosmic Ray Veto Detector \\
\hline T1044 & sPHENIX Calorimetry Tests \\
\hline T1048 & EIC PID \\
\hline T1037 & FLYSUB Consortium Tracking \& RICH \\
\hline T1018 & UCLA Spacordion Tungsten Powder Calorimeter \\
\hline T1042 & g-2 Straw Tracker Vacuum Tests \\
\hline T1073 & Precision Timing Profile Monitor \\
\hline T1072 & Muon Strips for Future Colliders \\
\hline T0979 & Fast Timing Counters for PSEC \\
\hline T1075 & Large Area ToF for ProtoDUNE \\
\hline T1034 & LArIAT: Liquid Argon in a Test Beam \\
\hline
\end{tabular}

Table TB-4: Test experiments performed in FY 2016. 


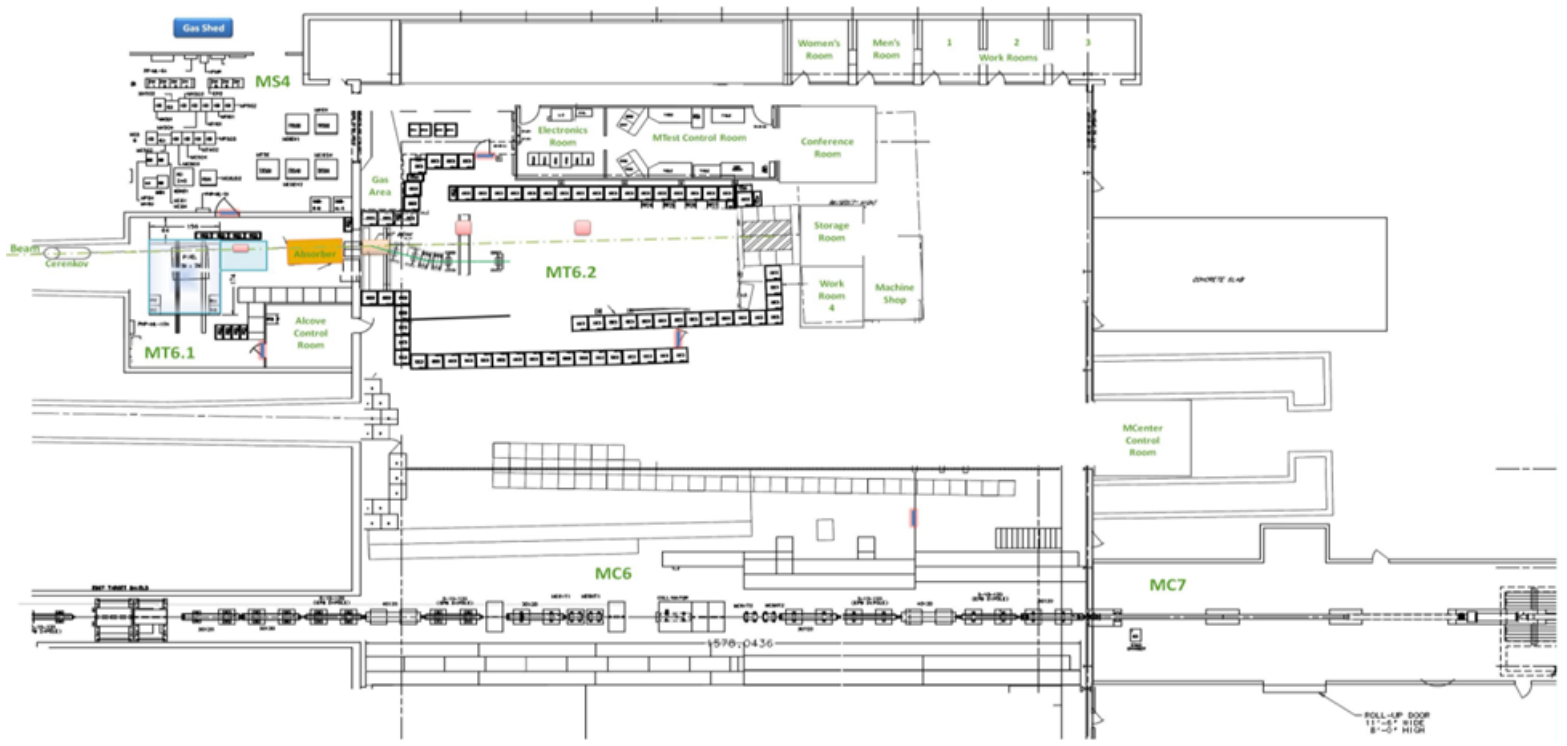

Figure TB-5: View of the Fermilab Test Beam Facility.

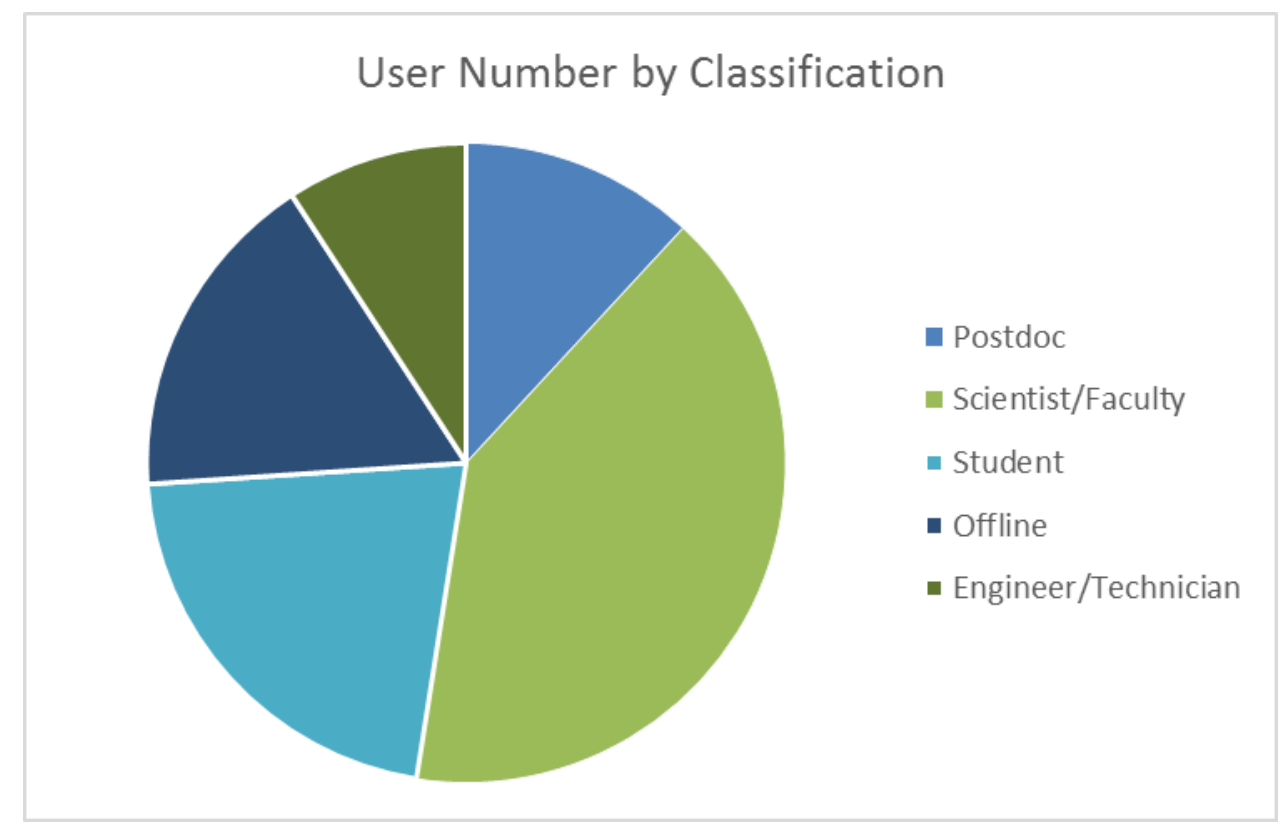

Figure TB-6: Number of users, broken down by classification. The total number of users for FY16 was 261 . Students include undergraduates and graduates. Engineers and Technicians refer to people that experiments brought with them so do not include technicians and engineers provided by Fermilab. Note that the number of experimenters using test beam data extends well beyond those who physically come to the facility. 


\section{Experiments by Research Focus}

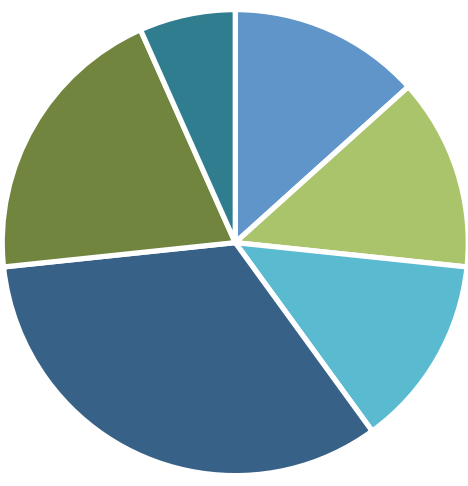

Neutrino

Muon

- Collider (LHC)

- Collider (Non LHC) - General R\&D

- Outreach/Facility

Figure TB-7: The research focus of the different groups that came to the test beam in FY2016. We support a broad range of users. 
FERMILAB-TM- 2640-DI

November 2016

\section{SECTION 2: \\ REPORTS FROM THE TEST EXPERIMENTS}


Report on Test Beam experiment T-979: Fast Timing detectors. (M.G.Albrow, E.Ramberg, S.Los; D.Ingram, C.Snyder, S. Durgut, M.Haytmuradov, V.Samoykenko)

Dates of Run: May $12-17^{\text {th }} 2016$ as secondary user, $120 \mathrm{GeV}$ protons.

Beam used: $120 \mathrm{GeV}$ protons

\section{Detectors under test: Quartz Timing Cherenkov (QUARTIC) detectors}

Goal: timing protons with $\sigma(\mathrm{t}) \sim 20-30 \mathrm{ps}$ in 20-channel array $(4 \times 5)$ of $3 \times 3 \mathrm{~mm}^{2}$ elements. Quartz radiator bars in form of $\mathrm{L}$ for light-guides to SiPM array.

Detector should be rad-hard and edgeless for operation close to LHC beam in Roman pots. This project is in R\&D. Presently diamond detectors are used for timing (worse resolution but more segmented and thinner,, so multiple layers possible).

Primary application: In CMS measuring $p+p \rightarrow p+X+p$ events.

Timing both protons, time difference kills pile-up at high luminosity $\sigma(\mathrm{t})=20 \mathrm{ps}$ gives $\mathrm{z}$ (collision) to $4 \mathrm{~mm} \mathrm{cf}$ $50 \mathrm{~mm}$ spread.

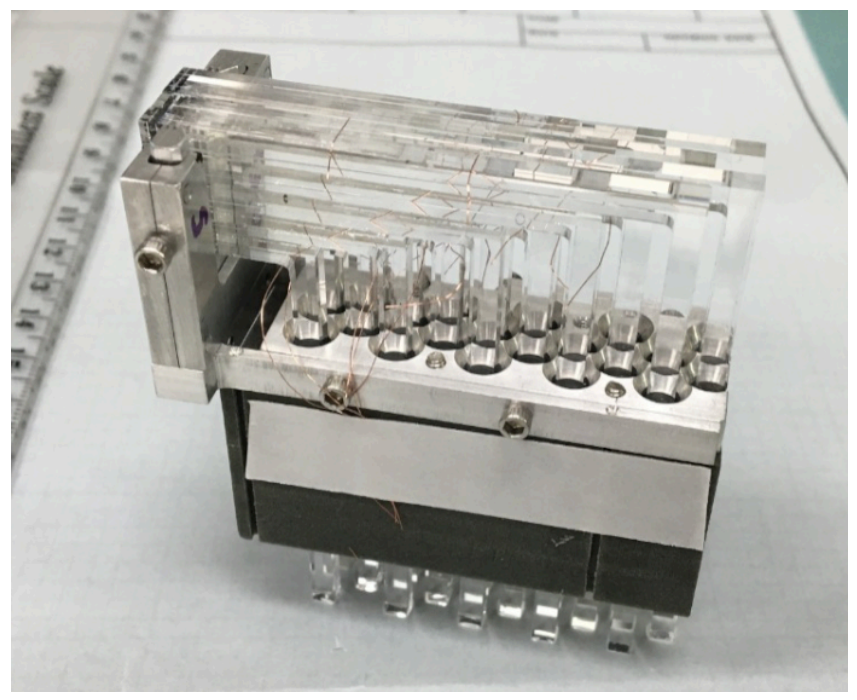

\section{Summary of conclusions:}

We had 30 ps resolution in an earlier prototype with two bars. Although these 20 bars are separated by 100 um there is too much light sharing between them, and the radiator bars should be aluminized or otherwise optically separated. This degraded the time resolution as we could only read out 3 bars at a time (in future should have 20 -channel readout). The time resolution from these tests was $\sim 60-80 \mathrm{ps}$. We have not yet published these results, but plan to do so (J.Inst). A further "upgrade" would be to replace the SiPM array with a micro-channel plate PMT (MCP-PMT). Further tests may be at CERN, in which case T979 can be considered completed. 
T-992 / Tests of radiation-hard sensors for the HL-LHC (R. Rivera, L. Uplegger)

Beam used: $120 \mathrm{GeV}$ protons

2015 Run dates: Oct 28-Nov 10, Dec 9-24

2016 Run dates: May 11-24, Jun 15-21

\section{Motivation and Goals:}

At the HL-LHC, after $2500 \mathrm{pb}^{-1}$ of data, the expected maximum fluence for the

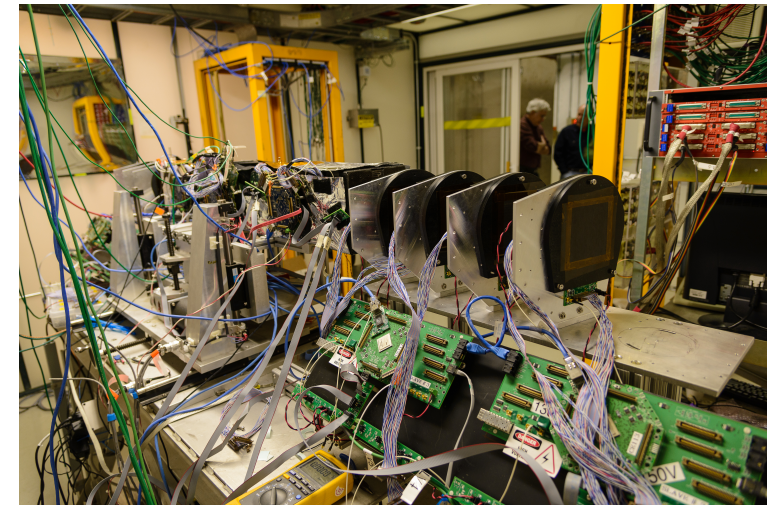

Figure 1 In situ T-992 test beam setup pixel region $(<20 \mathrm{~cm})$ will be $2.5 \mathrm{x}$ $10 \mathrm{E} 16 \mathrm{n}$ eq $/ \mathrm{cm}^{-2}$. To cope with this unprecedented radiation environment, there have been quite a few international collaborations formed to find possible solutions for vertex and tracking detectors at the HL-LHC. These include the RD42, RD49, and RD50 collaborations. A variety of solutions have been pursued. These include diamond sensors, 3D sensors, MCZ planar silicon detectors made from MCZ wafers, epitaxial, p-type silicon wafers and thin silicon detectors. The experimenters wish to compare the performance of this wide variety of detectors in a test beam before and after irradiation. To do so, the experimenters use the FTBF pixel and strip tracking telescopes which have $\sim 8 \mu \mathrm{m}$ resolution at the device under test. In particular, the experimenters are planning to study the charge collection efficiency of irradiated and un-irradiated devices and the spatial resolution as a function of the track incident angle. The experimenters will change the incident angle of the beam by moving the sensors, to investigate how the resolution varies with angle. Many physicists participating in this beam test are members of the RD42 and/or RD50 collaborations.

\section{Setup:}

The pixel and strip telescopes are read out through a custom DAQ system known as CAPTAN. A gigabit Ethernet board is used to route UDP data to a computer which is connected to a Fermilab server via internet. The readout boards are located close to the detector in the hut, and share a common clock and trigger signal. The detectors themselves may be operated up to $\sim 800 \mathrm{~V}$. No exposed HV parts are present.

\section{Results and Impact:}

The experimenters studied 3D silicon, a new prototypes of planar sensors with a small pitch $(25 \mu \mathrm{m})$ and several n-on-p type silicon thin planar sensors. 3D tracking detectors are 
promising radiation-hard candidates to replace planar detectors in the HL-LHC. Radiation damage effects are measured with regards to charge collection, efficiency, and resolution of the particle tracks in beam tests, as well as leakage current and pixel noise. We have studied two varieties of 3D sensors: 1E, 2E (the number referring to the number of implant pattern per pixel), irradiated at CERN with doses up to $4 \times 10 \mathrm{E} 15 \mathrm{n}_{\text {eq }} / \mathrm{cm}^{-2}$. After irradiation, the $2 \mathrm{E}$ showed the least degradation in efficiency and collected charge. These devices were provided by the Fondazione Bruno Kessler (FBK) in Italy. More work must be done to reach radiation hardness of $10 \mathrm{E} 16 \mathrm{neq} / \mathrm{cm}^{2}$ but right now the accumulated dose is limited by the readout chip that can't work anymore above $5 \times 10 \mathrm{E} 15 \mathrm{n}_{\text {eq }} / \mathrm{cm}^{-2}$.

We also measured the properties of new prototypes with a small pitch $25 \mu \mathrm{m}$, after they have been irradiated at CERN also up to $4 \times 10 \mathrm{E} 15 \mathrm{n}_{\text {eq }} / \mathrm{cm}^{-2}$. These prototypes worked well proving that it is feasible to make pixel detectors with pitches as low as $25 \mu \mathrm{m}$, without impacting significantly the performances at high-accumulated doses.

In December 2015 we also tested several prototypes of thin sensors with n-on-p technology which are right now the preferred candidate by the CMS collaboration. These devices were also fabricated at FBK and they performed well giving high efficiencies even if they only generate $1 / 3$ of the charge with respect to the traditional thicker sensors. We then irradiated them in February at Los Alamos and then retested in May 2016 at FTBF. 
T-1015 / Dual-readout calorimetry with heavy glasses ～(C.Gatto for the T-1015 Collaboration $^{1}$ )

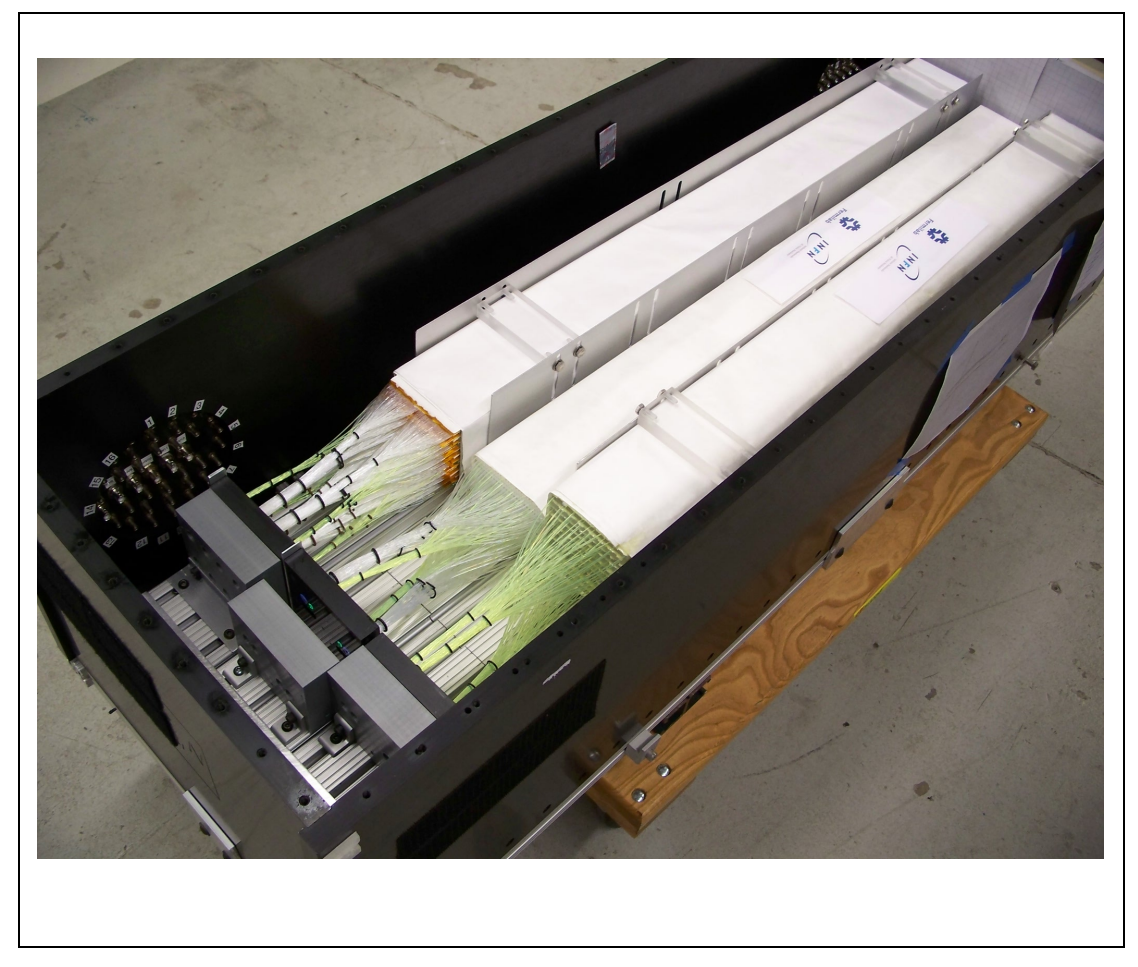

Figure 1: ADRIANO prototypes 2014-A, 2014B and 2015

Beam used: $120 \mathrm{GeV}$ protons, $32 \mathrm{GeV}$ muons, secondary from $0.5 \mathrm{GeV}$ to $12 \mathrm{GeV}$

Run dates: Nov 11, 2015-Dec 27, 2015

\section{Motivation and Goals:}

The ADRIANO technology (A Dual-readout Integrally Active Non-segmented Option) is extensively being developed as part of the research program of T1015 Collaboration for experiments at future lepton colliders or at fixed target with high intensity beam. [1] New construction techniques have been exploited and several detector prototypes have been assembled and tested at FTBF since the inception of T1015 Collaboration to study their performance. The ADRIANO, technology, initially devised to improve the performance of hadron calorimeters through the mechanism of dual-readout compensation, has been extended for applications to electromagnetic calorimeters. The fast response of ADRIANO, along with its intrinsic particle-id features, make this technology particularly well suited for future High Intensity frontier experiments.

\footnotetext{
${ }^{1}$ https://web.fnal.gov/experiment/FTBF/TSW\%20Library/T1015_mou.pdf?Web=1
} 


\section{Setup:}

1. ADRIANO 2014A, 2014B and 2015 prototypes

2. Three fingers for triggering the beam

3. FEB from nu2e experiment

The FTBF facilities used during the experiment are listed:

1. Pick-up for the transportation of the detector to F-Test

2. Crane for the installation of the detector

3. Remotely controlled moving table $2 \mathrm{~B}$

4. Remotely controlled rotating table

5. One wire chamber

6. Cherenkov based PID system

7. HV power supply and distribution systems (COW).

\section{Results and Impact:}

Light yield has been measured for a total of four ARIANO prototypes. The values obtained range from 280 p.e./GeV (for the ADRIANO 2015 detector) to 340 p.e./GeV (for the ADRIANO 2014A detector). These values meet the minimum requirement for use of ADRIANO in high precision experiments at lepton colliders (i.e ILC or CLIC). The results have not been shown to any conference yet. 
FERMILAB-TM- 2640-DI

November 2016

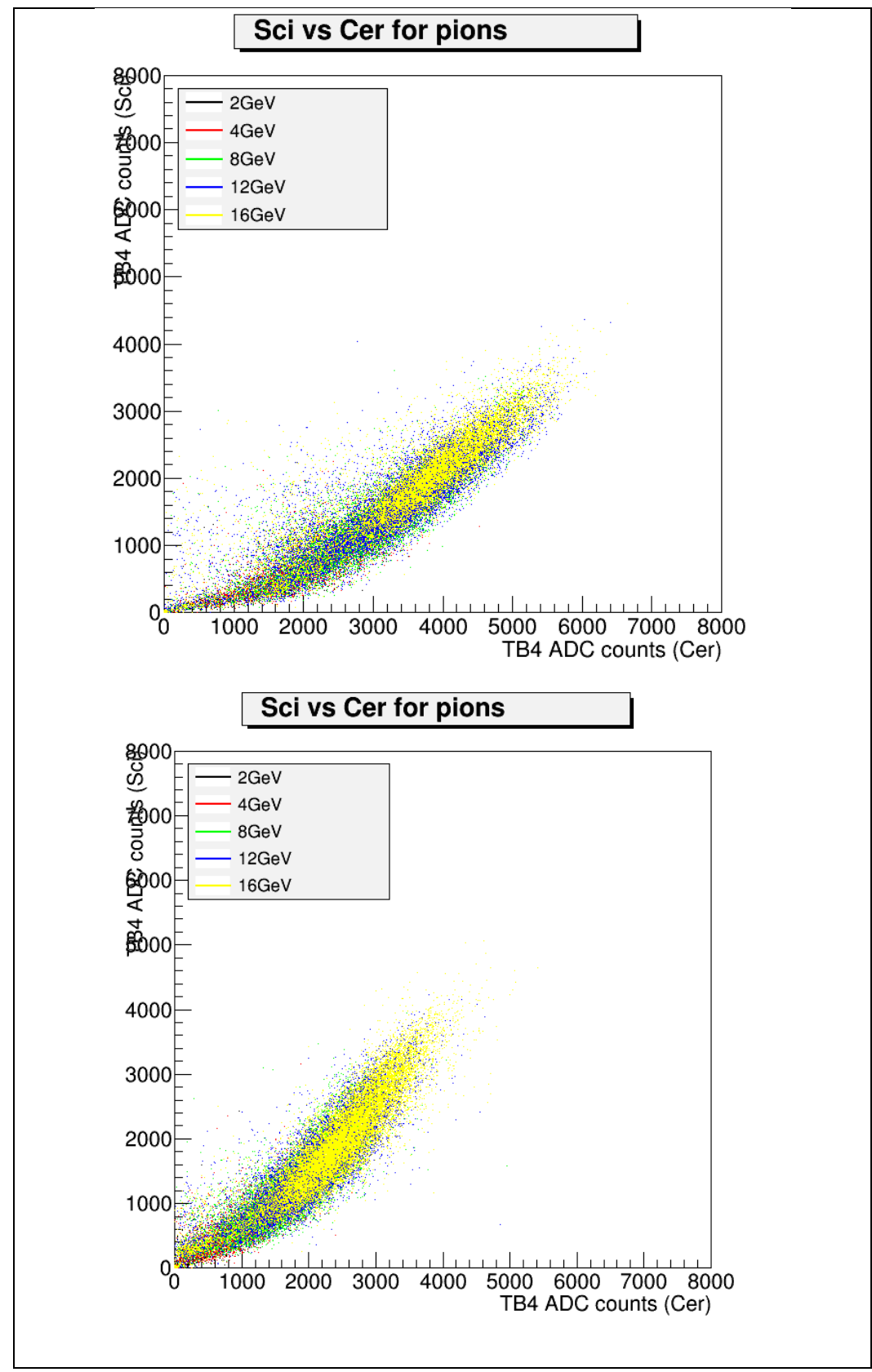

Figure 2: Scatter plots of scintillation vs Cerenkov signals for ADRIANO 2014A and 2014B detectors 
T-1018 / (R. Esha ${ }^{1}$, A. Kisilev², O. Tsai ${ }^{1}$, L. Wen ${ }^{1}$ )

Beam used: $1 \mathrm{GeV}-16 \mathrm{GeV}$ electrons

Run dates: May 4 -May 11

Motivation and Goals:

We are (eRD1 calorimeter consortium) continuing development of $\mathrm{W}$ powder ScFi calorimetry technology for Electron Ion Collider (EIC) experiments. Our main goal for 2016 was to perform a decisive test for a high-resolution EMCal built with our technique for the outgoing electron direction for an EIC detector. Test run 2016 is continuation of previous year efforts. In 2016 we tested re-worked prototype tested in 2015 along with new prototype build with square fibers (depicted in Fig. 1).

A decisive test provided answers to these questions:

- Is production homogeneity of the blocks (+- $0.2 \%)$ sufficient?

- Are local density/composition variations under control? (Especially for the W/Sn composite absorber during packing.)

- Is the light yield sufficient to allow for compact readout with Si sensors in the future?

- What is the effect of the 'dead' areas within and between super-blocks?

- What are benefits of using square fibers?

\section{Setup:}

We used the MT6.2-B area for the experimental setup. Instrumentation provided by the test beam facility included a Lead Glass calorimeter, movable table 2B, alignment laser system, Cerenkov counter, CAMAC and NIM crates, some signal cables, patch panels, cameras and helium tubes to reduce multiple scattering. In addition we used the MWPC tracking system as a stand-alone system for monitoring beam conditions (not in our data stream).

\section{Results and Impact:}

The energy resolution of the new EMCal prototype is shown in Fig. 2. It is much better $(\sim 30 \%$ better $)$ then what was achieved in 2015 . The test results for the new detector are very promising. The uniformity and energy resolution of this detector are already slightly better than that of one of the best forward fiber calorimeter of the past, the excellent $\mathrm{H} 1$ EMCal. Future improvements need to be made to reduce some dead layer between EM blocks. Contributions to the constant term from mechanical imperfections $(\sim 1.5 \%)$ quite 
low already but must be kept as small as possible, because there will be additional contributions to this constant term in the future from compact readout and calibrations. The design goal for EIC with the new prototype was reached with PMT readout.

ECal Resolution

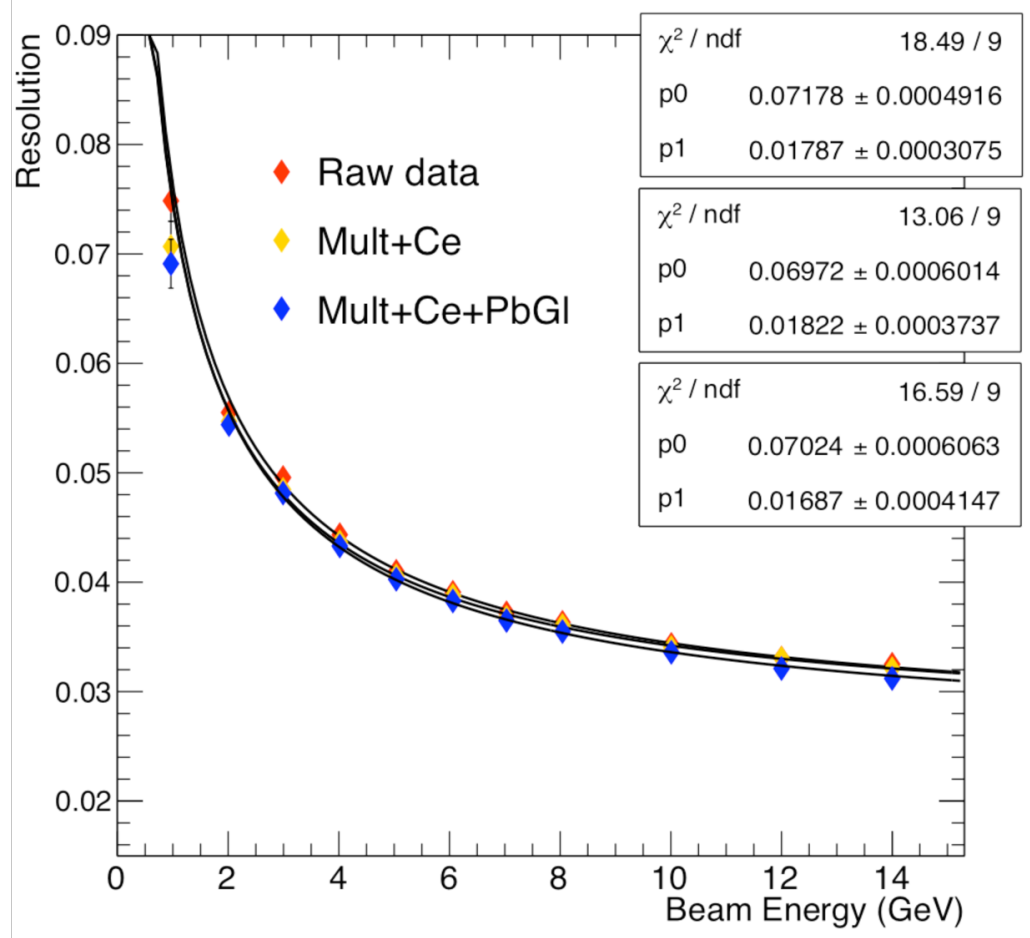

Figure 2: Energy resolution for new EM W/ScFi (square fibers) Prototype.

Results of the T1018 2016 test run were presented in XVIIth CALOR conference on May 16, 2016 by C.M. Camacho (CNRS/IN2P3 (France)) for eRD1 calorimeter consortium, with proceedings to follow. 
T-1034 / LArIAT (F. Cavanna, J. L. Raaf)

Beam used: $8-60 \mathrm{GeV} \mathrm{p}^{+/-}$

Run-I dates: May 1 - July 3, 2015

Run-II dates: February 19 - August 11, 2016

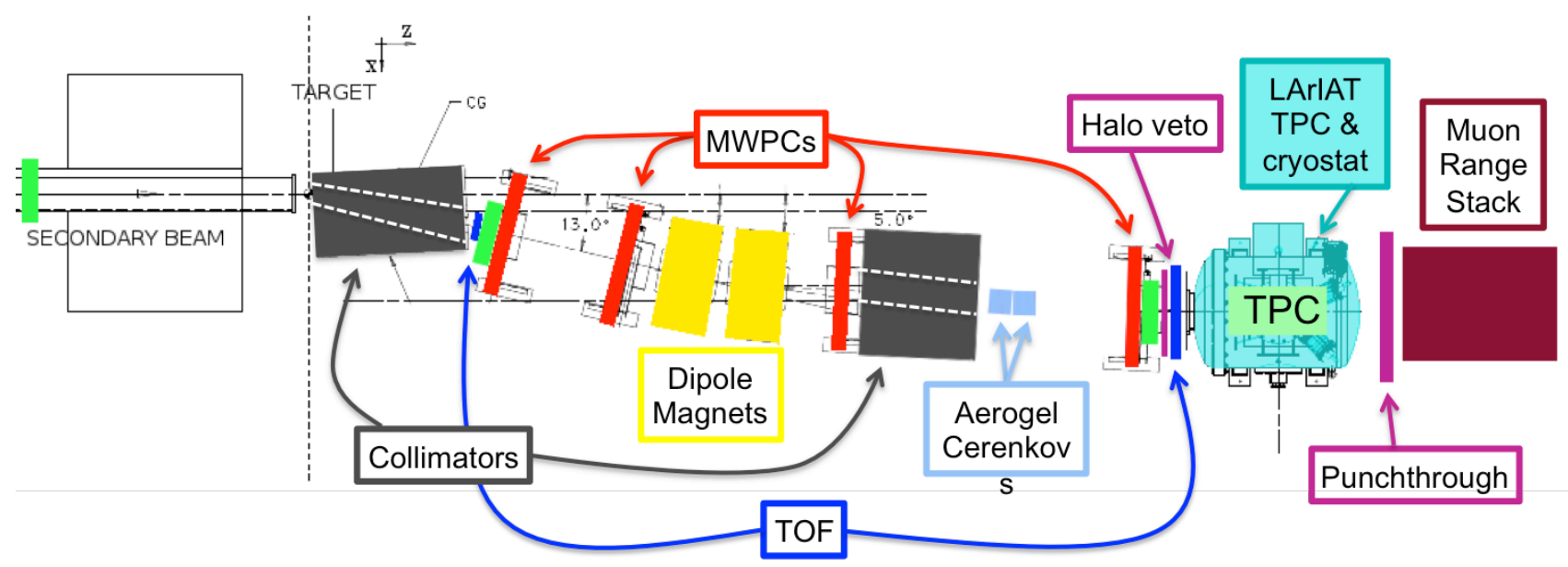

Figure 1: LArIAT layout in MCenter beamline

\section{Motivation and Goals:}

LArIAT (T-1034) is characterizing the performance of liquid argon time projection chambers (LArTPCs) in the range of energies relevant to short- and long-baseline experiments such as MicroBooNE, the SBN program, and DUNE. Measurements made in LArIAT aim to be useful for both neutrino physics and for proton decay searches. Measurements of charged pion and kaon interaction cross sections will be made, and these data will also be used to optimize particle identification capabilities. In addition, LArIAT will study energy resolution improvements that may be achievable by combining information from scintillation light and ionization charge signals. The data collected in LArIAT will also be used to study the possibility of determining the sign of charged particles (pions/muons) without a magnetic field. 


\section{Setup:}

The LArIAT setup is shown in

Figure 1, located in the MCenter beamline at MC7. The incoming secondary beam was operated in both positive and negative polarity for a range of beam energies, and the tertiary magnets were also operated across their full range. The setup uses 4 multiwire proportional chambers (MWPCs) and 2 time-of-flight (TOF) scintillator paddles provided by FTBF. Additional beam instrumentation, such as aerogel Cherenkov counters, halo and punchthrough veto scintillator paddles, and muon range stack were put in place by the experiment. The heart of the experiment is a two-plane ( 240 wires each, $4 \mathrm{~mm}$ spacing) liquid argon time projection chamber (LArTPC) with 2 cryogenic PMTs and 3 SiPMs viewing the active volume.

\section{Results and Impact:}

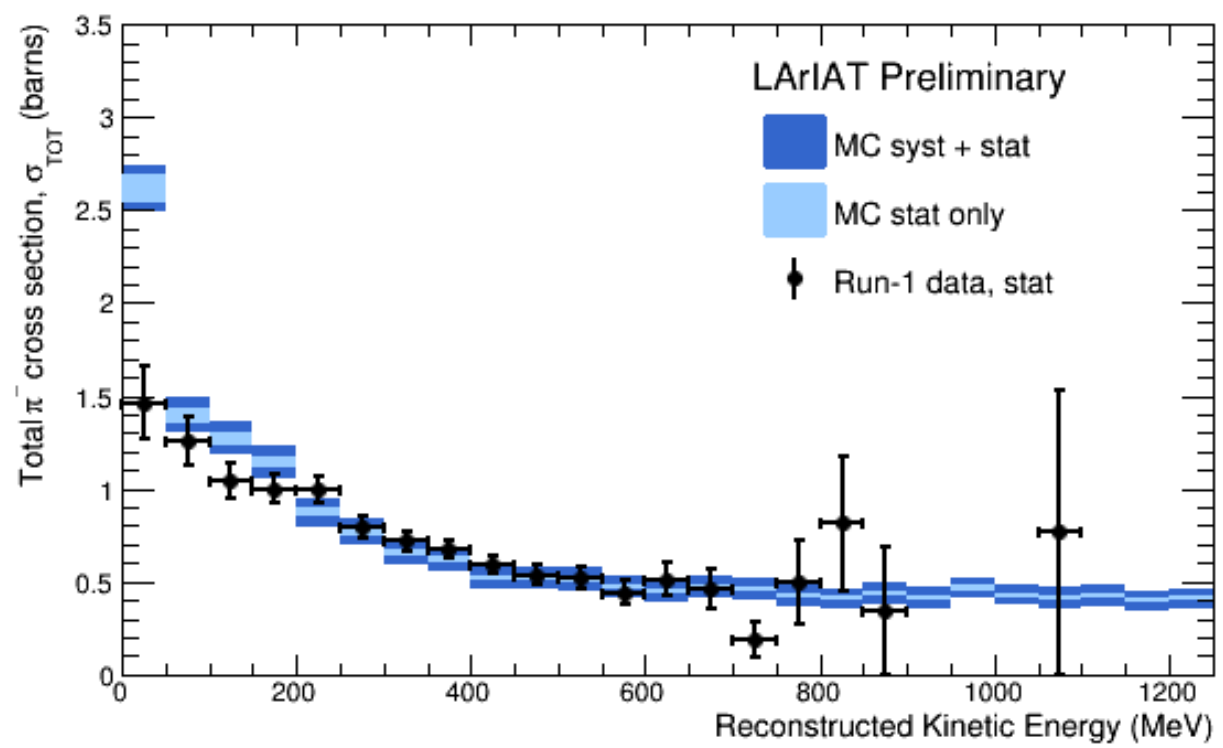

Figure 2: Preliminary results for the pion-argon total cross section using LArIAT Run-I data.

LArIAT reported its first total pion cross section results at a Fermilab Wine \& Cheese (Joint Experimental-Theoretical Physics) Seminar on April 8, 2016. This analysis applies fully automated 3D reconstruction techniques to the negative polarity Run-I data (collected in 2015). We select pions from the mix of particles that arrive at the LArTPC, making use of data from the wire chambers and time-of-flight counters to separate particle types in the 
beamline, and to measure the entering particle momenta. This is the first ever measurement of the total pion-argon cross section. A paper is in preparation, to be submitted to a peer-reviewed journal such as Phys. Rev. D.

Analyses of the cross sections for exclusive pion interaction channels are currently underway as well, including pion charge exchange, pion absorption, and pion scattering.

We are also currently analyzing data with an aim to measure the kaon interaction cross sections (total and exclusive channels). Our beam configurations in Run-II (2016) were chosen to enhance the number of collected kaons, and so these analyses have begun with the Run-II data. We estimate that we have roughly 1000 kaons in this dataset.

Each of the cross section measurements will be useful for improving and validating Geant- 4 simulations, which can then be used for all future LArTPC experiments. These data will also be used to refine particle identification techniques in LArTPCs. The results will feed back to future LAr experiments, where a better understanding of the capabilities and efficiencies for identifying different particle species will lead to improved measurements of neutrino interactions. 
T-1037 / Flysub (TK Hemmick, B. Azmoun, A. Zhang)

Beam used: Many from $8 \mathrm{Gev}-120$ $\mathrm{GeV}$

Run dates: April 2016

Motivation and Goals:

The detector to be studied in this test is a combination Time Projection Chamber (TPC) and a Cherenkov detector. It consists of a

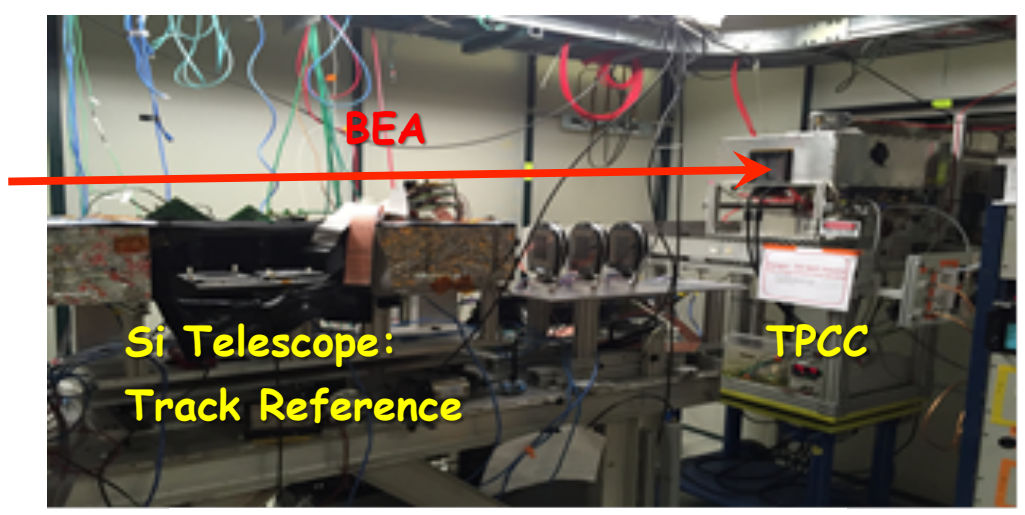
small TPC with a $10 \times 10 \times 10 \mathrm{~cm} 3 \mathrm{drift}$ volume that is read out with a multistage GEM detector, along with a second $10 \times 10$ Figure 1 T1037 Setup. $\mathrm{cm} 2$ photosensitive GEM detector equipped with a CsI photocathode to detect Cherenkov light produced in the TPC gas volume. This detector could be used to provide tracking information and particle ID in a single detector and could be used in a variety of applications at a future Electron Ion Collider (EIC). The goal of this test is to study the tracking performance of the TPC portion of the detector and the light detection capabilities of the Cherenkov detector, both individually and in combination with each other. The tracking portion of the detector is similar to the Minidrift GEM detector tested in the 2013 FLYSUB test and the Cherenkov portion is similar to the RICH detector tested in those same tests.

\section{Setup:}

The test was set up in the upstream clean tent immediately behind the FTBF silicon tracker. Data was taken triggered on the triple coincidence of three silicon modules from the Silicon Telescope at the test beam.. Triggers were sent simultaneously to the T1037 DAQ and to the silicon tracker DAQ. These two systems recorded events to separate files and these events were combined in offline analysis. Much of the tune-up data was taken with 120 $\mathrm{GeV}$ protons at $10 \mathrm{k}$ protons per spill since this was the beam desired by others with whom we shared the beam time. The most precious data, however, used an energy of $12 \mathrm{GeV}$ since this energy had pions/electrons above our Cherenkov threshold and kaons/protons below threshold. In this way we were simultaneously able to measure tracks that should or should not fire the Cherenkov counter. To facilitate the analysis, we tuned the FTBF Cherenkov detector so that its threshold matched our device and thereby we were able to use it to separate out data into two samples and compare the Cherenkov response. 

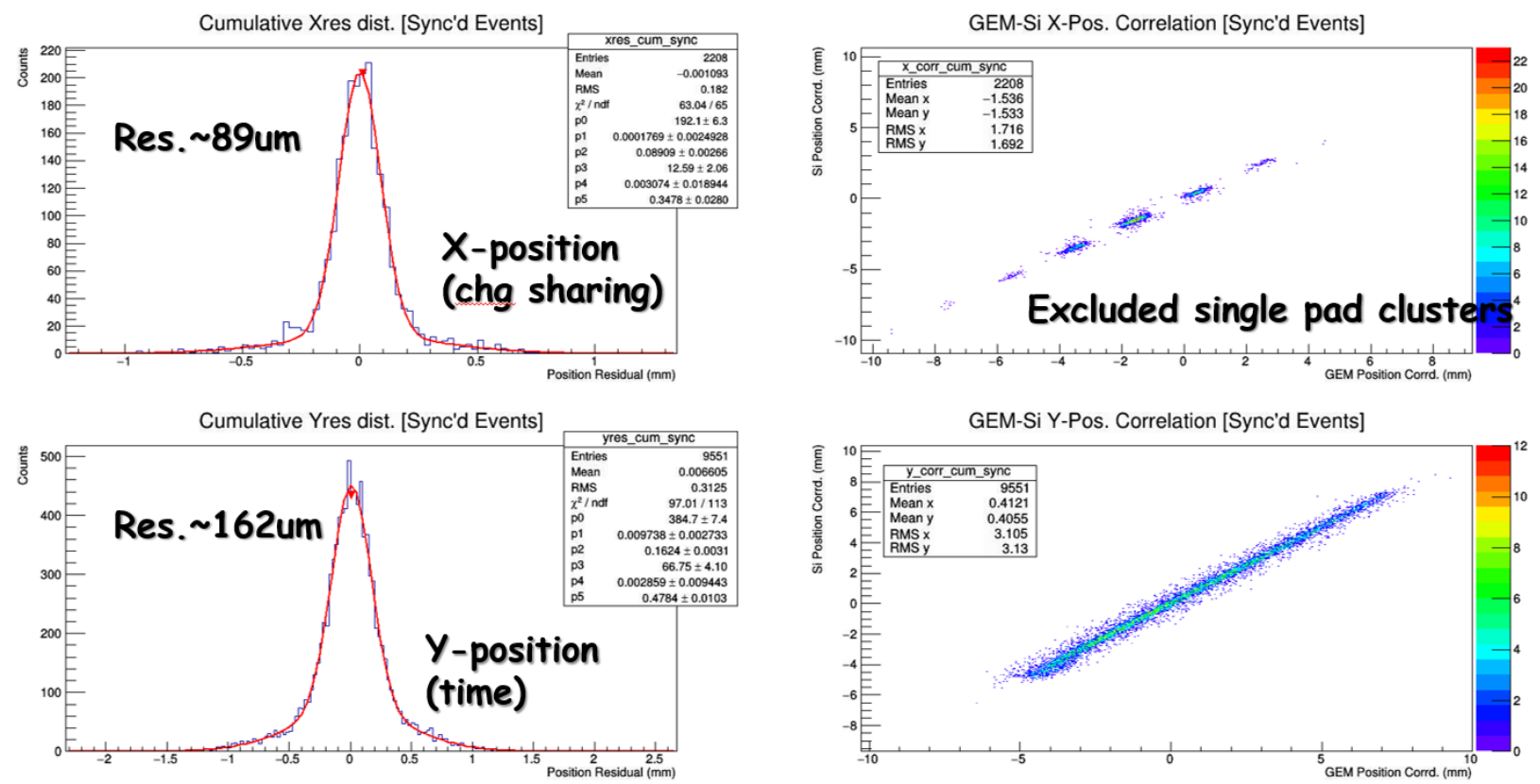

Figure 2 The top row is the TPC $x$-coordinate (measure3d by charge sharing) and the bottom row is the TPC $y$ coordinate measured by drift times. The right column shows the correlation with the FTBF silicon and the left column shows the residuals.

\section{Results and Impact:}

We consider the beam test to have been a complete success. We saw tracks in the TPC and cherenkov hits in the HBD section. Figure 2 shows the resolution of the TPC system as determined by comparison with the FTBF silicon detector. The result is absolutely suberb, especially considering this is the first time that a TPC has been operated with pure $\mathrm{CF}_{4}$ as its working gas.

Figure 3 shows the bias scan of the drift field of the cherenkov section of the detector. At positive bias the cherenkov section receives BOTH ionization from the primary track as well as cherenkov light. At negative bias, the detector is sensitive to only cherenkov light. Using the known specific ionization of MIPS in $\mathrm{CF}_{4}$ we are thereby able to calibrate the plot to learn that our cherenkov signal was 10 photo-electrons. This is a phenomenal signal since the prototype detector was less than $1 / 2$ the length of our final device. This makes our device among the brightest (as measured by $\mathrm{N}_{0}$ ) cherenkov detectors ever constructed. One should note, however, that an HBD-style cherenkov device does intercept a small ionization signal that we were also able to meaure in the experiment.

Figure 4 shows in blue the pulse height of the cherenkov detector for particles that are above our cherenkov threshold (as independently determined by the FTBF beamline cherenkov devices). In red is the response for particles that are below threshold. It should be noted that the prototype has less than $1 / 2$ the length of the full device. This means that 
the response of the full device would be $2 \mathrm{X}$ higher for the blue curve, but exactly the same for the red curve. We conclude that the device eID performs exactly as our best hopes.

\section{MIP Signal Vs Drift Field}

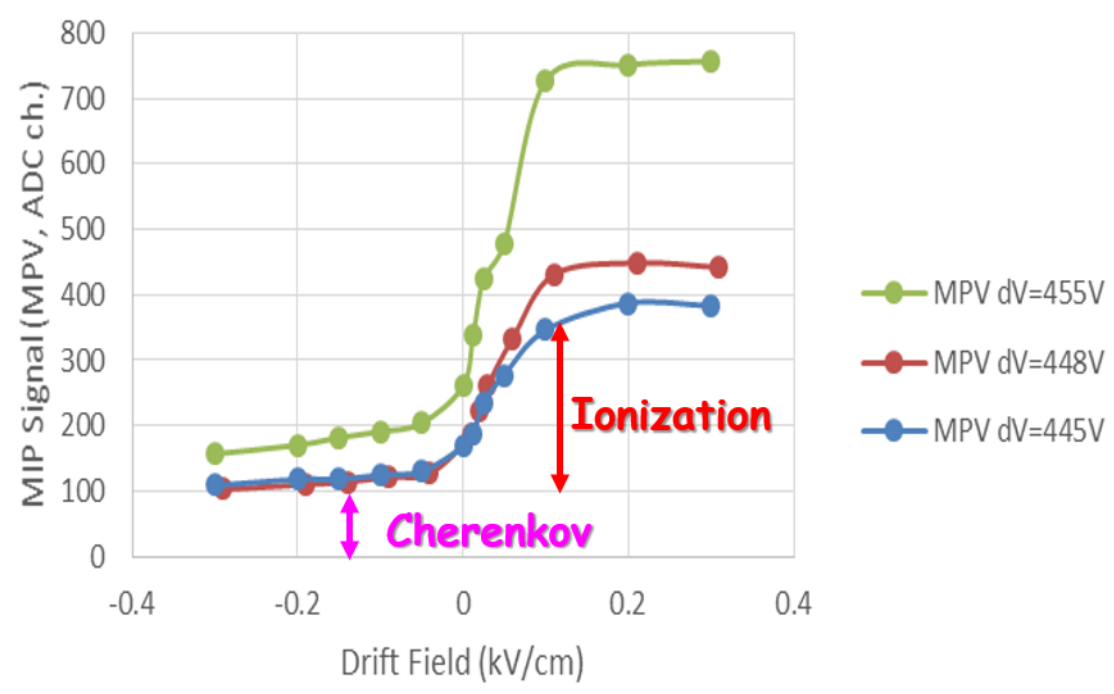

Figure 3: Bias scan of the cherenkov detector.
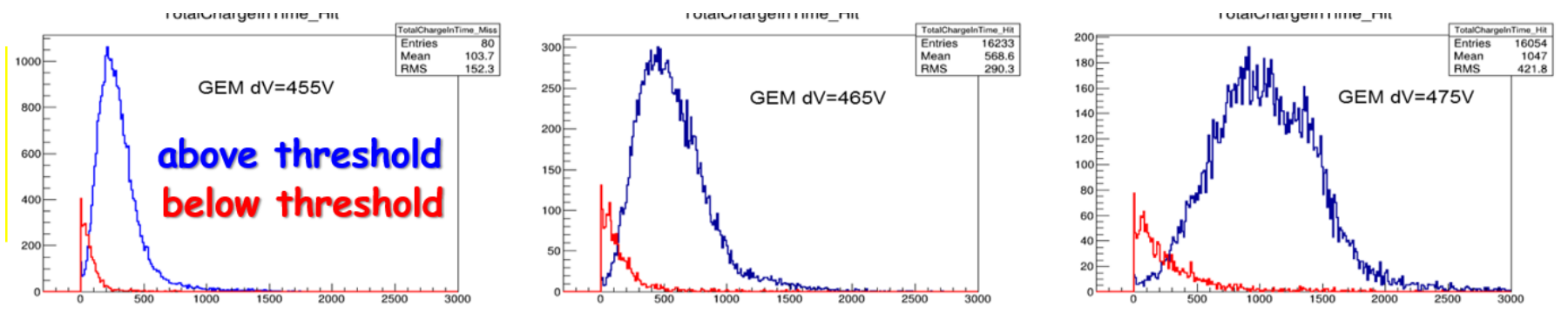

Figure 4: Response to particles above (blue) and below (red) threshold. Each plot is a different detector gain.

These results were first shown at the RICH 2016 conference in Bled Slovenia on September 5,2016 . They were exceptionally well received and provoked much thought. Our device is the first ever to simultaneously record the trajectory (with high precision), specific ionization (for $\mathrm{dE} / \mathrm{dx}$-based hadron ID), and cherenkov light (for eID) from a single gas volume. This technique can dramatically reduce the size and thereby cost of future collider experiments by combining all this functionality into a single volume, reducing the physical size of the full detector.

We are currently preparing a publication that we expect to submit in either October or November 2016. 


\section{T-1041 / Radiation-Hard Scintillators （B. Bilki, Y.Onel)}

Beam used: primary protons

Run dates: October 21 - November 3, 2015

\section{Motivation and Goals:}

Developing radiation-hard scintillators for future and upgrade collider experiments. Tests of intrinsically radiation-hard, commercial scintillator performances (examples are Polyethylene Naphthalate, PEN, and Polyethylene Terapythalate, PET); and novel developments of scintillator materials (Iowa Scintillator-X).

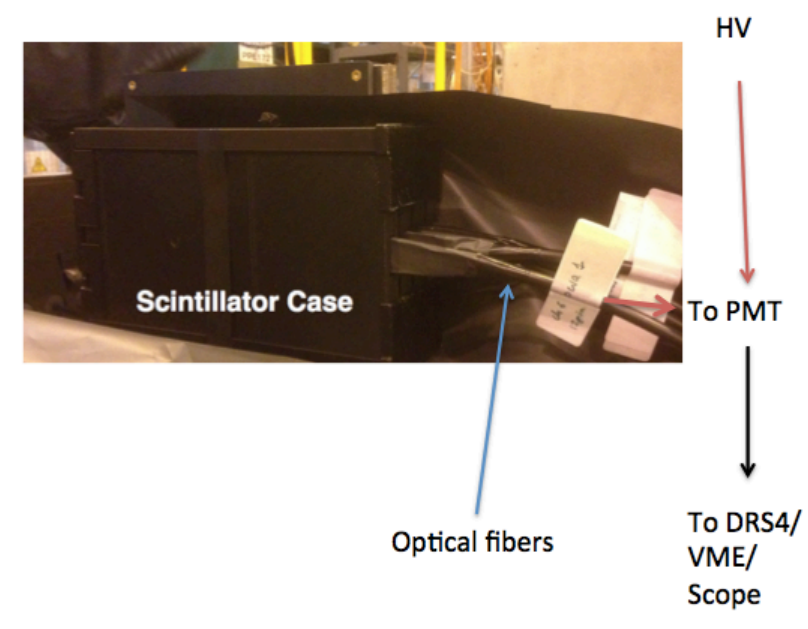

\section{Setup:}

The test setup includes single layers of scintillators in a light-tight cassette structure where different scintillators are tested one at a time. Multiple tiles of the same scintillator in the cassette structure are also tested. The readout is either with a directly coupled photodetector (SiPM) or with a wavelength shifting fiber placed inside the grooves in the scintillator tiles, which are then coupled to a photodetector outside the cassette.

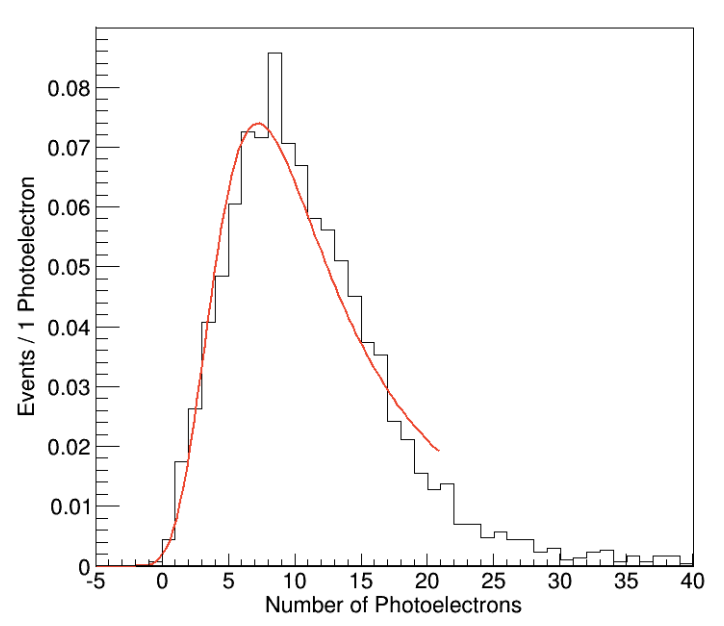

\section{Results and Impact:}

The recent development of a radiation-hard scintillator, Scintillator-X, was tested for the first time. Top figure shows the response of a 2 $\mathrm{cm} \times 2 \mathrm{~cm} \times 0.6 \mathrm{~cm}$ tile read out with a photodetector directly coupled to a polished edge (other sides/edges are not polished and no reflective wrapping was used). A most probable value around 7 photoelectrons was measured for MIPs. The full width of the pulse at $1 / 10$ amplitude was $15 \mathrm{~ns}$. A patent application was filed with the University of

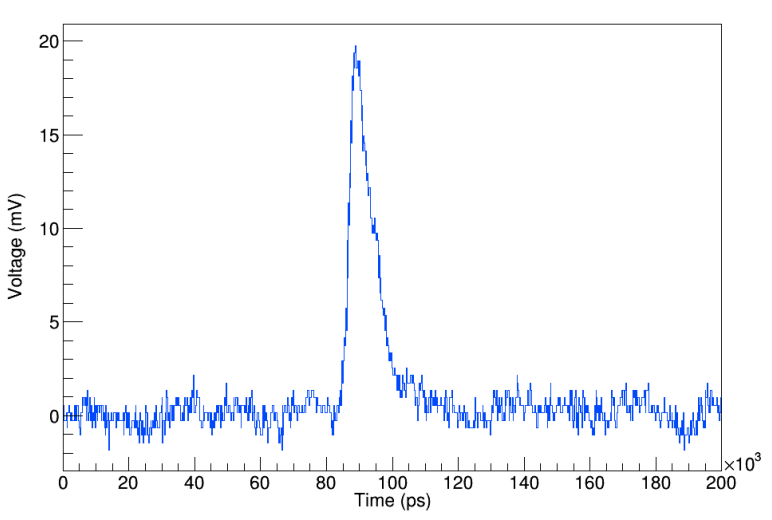
Iowa. 


\section{T-1041 / CMS High Granularity Calorimeter (B. Bilki, J. Freeman)}

Beam used: primary protons, secondary beams at various energies

Run dates: March 23 - April 5, June 22 - June 28, July 13 - July 19, 2016

\section{Motivation and Goals:}

The CMS Experiment of the LHC is going towards a Phase II Upgrade where the endcap calorimeters will be replaced with a High Granularity Calorimeter (HGC). The first beam tests of the HGC were performed at FTBF.

\section{Setup:}

Several layers of Silicon active layers interleaved with Tungsten absorbers are placed in a hanging file structure. The data is digitized with on-detector custom readout electronics. For certain runs Silicon trackers and downstream detectors were used for tracking, timing and particle identification.

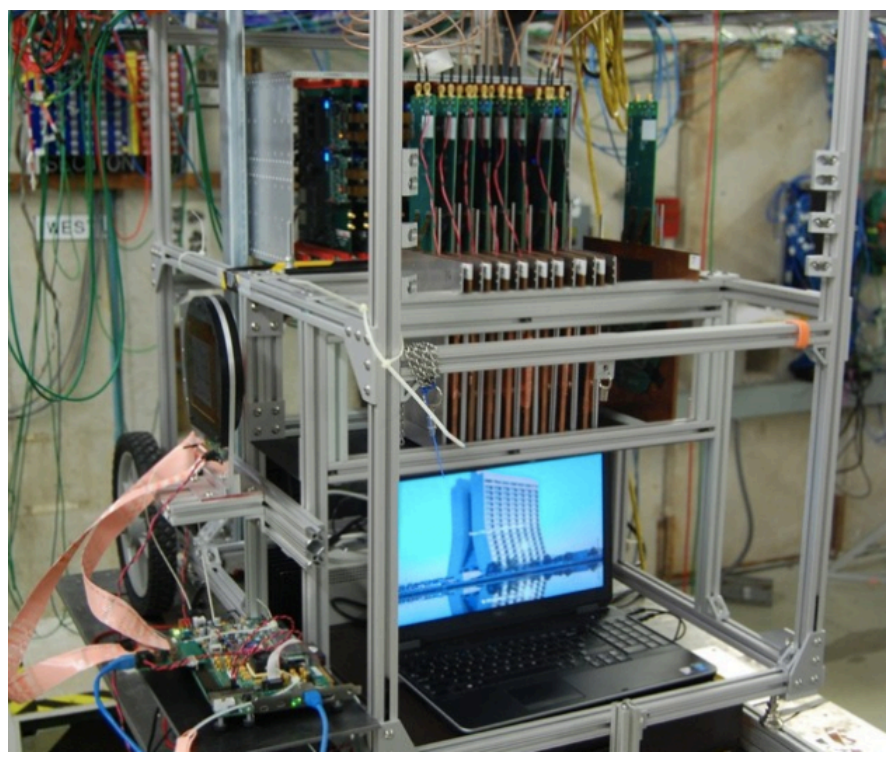

\section{Results and Impact:}

The first tests of the HGC were completed successfully. Figure shows the longitudinal shower shapes for electrons at various energies. These first measurements resulted in many conference talks.

A larger scale prototype is in progress steered by the results obtained at FTBF.

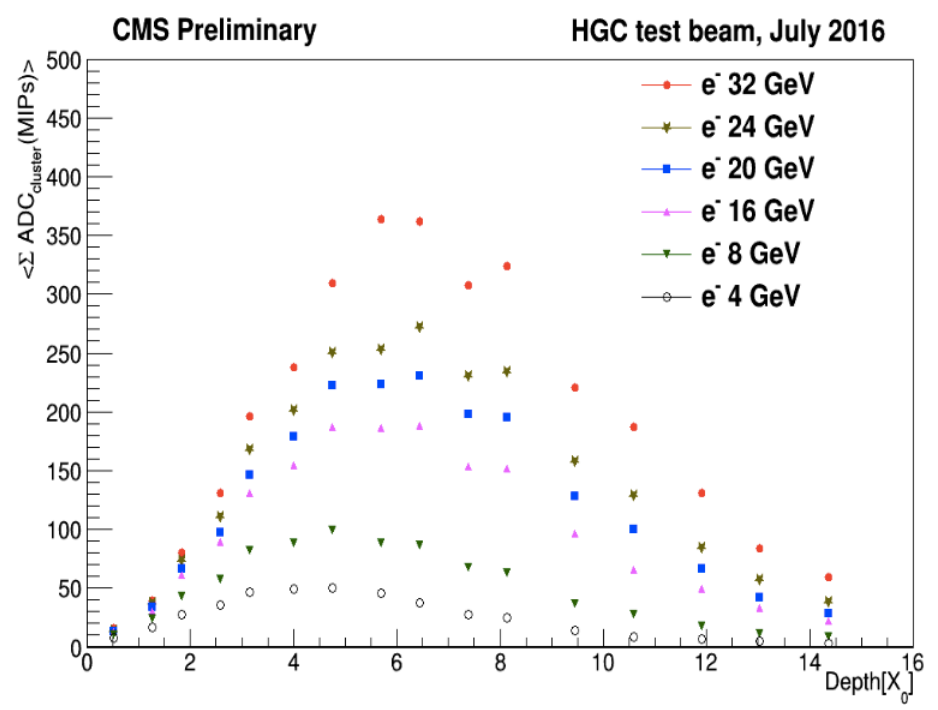




\section{T-1041 / RPC Rate Tests （B. Bilki, J. Repond)}

Beam used: primary protons

Run dates: May 18 - June 7, 2016

\section{Motivation and Goals:}

Developing high-rate Resistive Plate Chambers (RPCs) with low-resistivity glass and tests of novel single-glass RPCs. The low-resistivity glasses are either commercial or custom development by the Iowa Coe College.

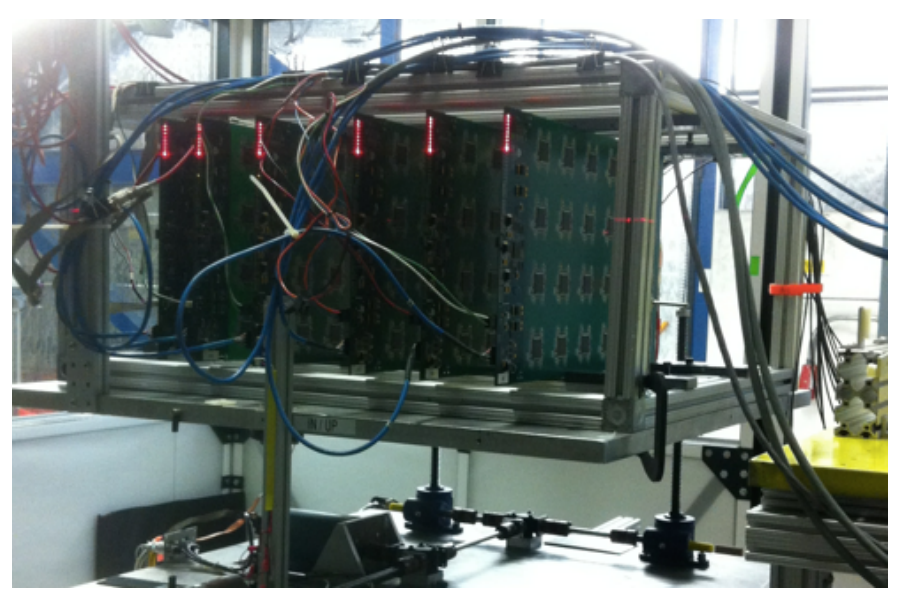

\section{Set up:}

The test setup includes six layers of RPCs constructed with different glasses. They are read out by the CALICE Digital Hadron Calorimeter readout boards simultaneously. The beam rate is adjusted gradually up to the machine limits.

\section{Results and Impact}

The recent developments on the lowresistivity glasses show about an order of magnitude improvement on the rate capability. The crucial point is that these glasses are developed in home, and the resistivity can be fine tuned. The novel 1glass RPCs work successfully and result in almost unity pad multiplicity.

Results of these tests were published in two journal articles:

- N. Johnson et.al., "Electronically Conductive

Vanadate Glasses for Resistive Plate Chamber Particle Detectors", International Journal of Applied Glass Science, 6 [1] 26-33, 2015. - M. Affatigato, et. al., "Measurements of the Rate Capability of Various Resistive Plate Chambers", JINST 10 P10037, 2015.
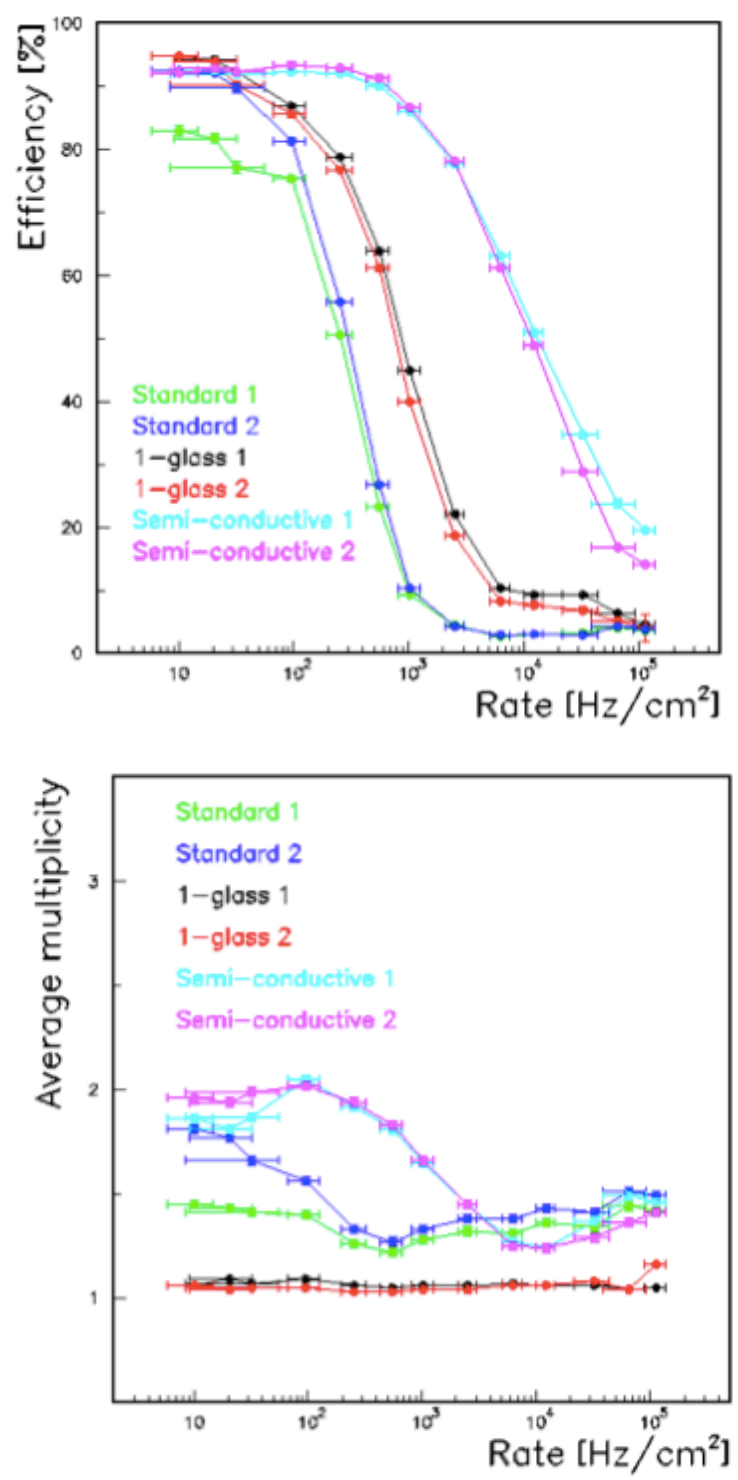
T-1042 / G-2 (J. Price, B. Casey)

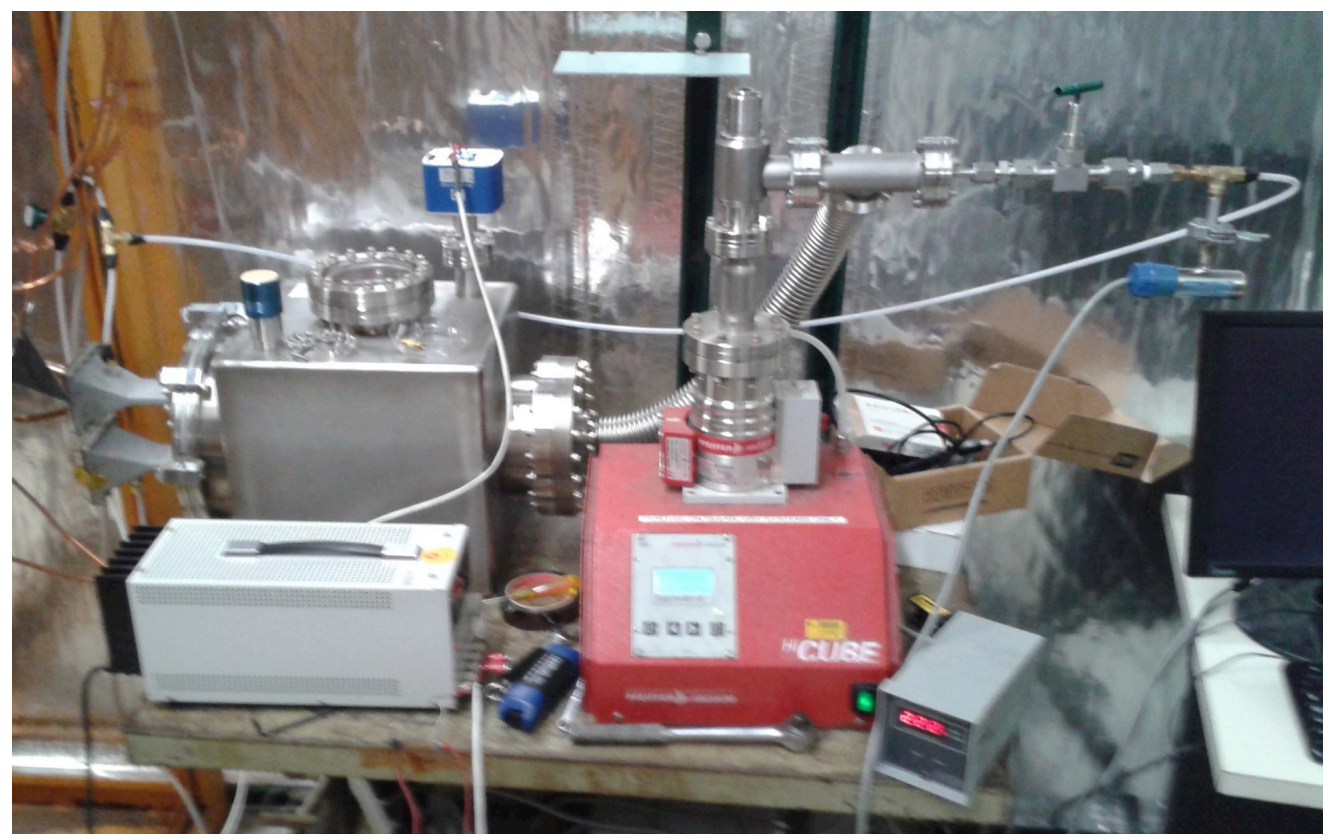

Figure 1: Setup at $m$-test in the gas room. The module is placed inside the vacuum tank and pumped down using the Pfeiffer turbo vacuum pump. The gas is supplied from MTest.

\section{Beam used: No}

Run dates: July $1^{\text {st }} \rightarrow$ July $20^{\text {th }}$, August $22^{\text {nd }} \rightarrow$ August $31^{\text {st }}$

\section{Motivation and Goals:}

The aim of this test is to measure the leak rate of a single module from the straw trackers to be used in the g-2 experiment for different gases, namely nitrogen, carbon dioxide and argon ethane. The leak rate for a single module must be kept low enough for the required vacuum. Before the module assembly the leak rate of the straws is measured using $\mathrm{CO}_{2}$, but in the experiment $\mathrm{Ar}-\mathrm{C}_{2} \mathrm{H}_{6}$ will be used, therefore an accurate measurement of the ratio of the leak rates using these two gases is required.

\section{Setup:}

The module was placed inside the vacuum tank, and connected up to the gas supply. The supply is always setup with nitrogen available, which is used to clean the system, and an alternative gas, which alternated between $\mathrm{CO}_{2}$ and $\mathrm{Ar}-\mathrm{CO}_{2} \mathrm{H}_{6}$. The turbo vacuum pump is connected to the vacuum tank, and also the straws, since measurements are also required when there is a vacuum in the straws in order determine the out-gassing of the module. A valve was placed on the gas input and output to isolate the system when pulling vacuum on 
the straws. To perform the rate of rise tests the vacuum pump valve is closed and the pressure inside the vacuum tank is read out by the connected gauge at a rate of $1 \mathrm{~Hz}$ for 600 seconds. This test was repeated for a second module in late August.

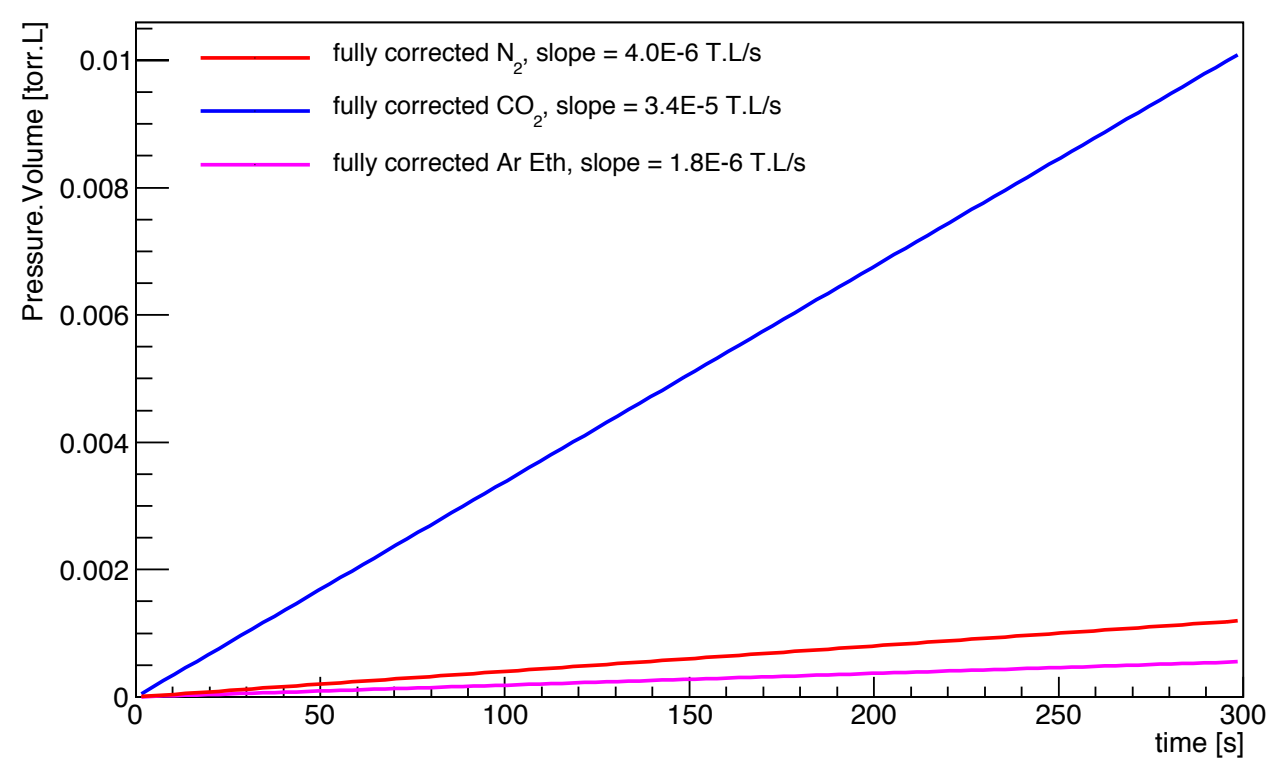

Figure 2: The average rate of rises for the different gases, after corrections for the background and gauge reading have been applied.

\section{Results and Impact:}

The results are shown in figure 2, where the rate of rises have been corrected for both the background out-gassing and the gas correction factors for the gauge. The leak rate through the straws for Carbon dioxide was determined to be a factor of 20 higher than for ArgonEthane.

Extrapolating to a full tracker station, which contains 8 modules, it was determined that the leak rate required for the straws before being used in the modules (determined using $\mathrm{CO}_{2}$ ) could be increased by a factor of 3 and we would still be able to achieve the desired vacuum in the experiment. 


\section{T-1043 / Mu2e CRV Scintillation Counters （Steve Magill for the CRV Group)}

Beam used: $120 \mathrm{GeV} \mathrm{p}$

Run dates: Feb 17-Mar 1, Jun 8-14, 2016

\section{Motivation and Goals:}

Several configurations of prototype scintillation counters for the Mu2e Cosmic Ray Veto (CRV) detector were tested. The 3 meter long counters were made of extruded plastic scintillator and contained 2 wave length shifting (WLS) fibers readout by SiPMs on both ends. The main goal was to measure the photoelectron (PE) yield to test whether the light yield met the required specifications ( $25 \mathrm{PE} / \mathrm{cm}$ at a position $1 \mathrm{~m}$ from the readout) for the CRV. XY and angular scans and data from counters with different fiber diameters and scintillator mix were taken to study variations in the PE yield.

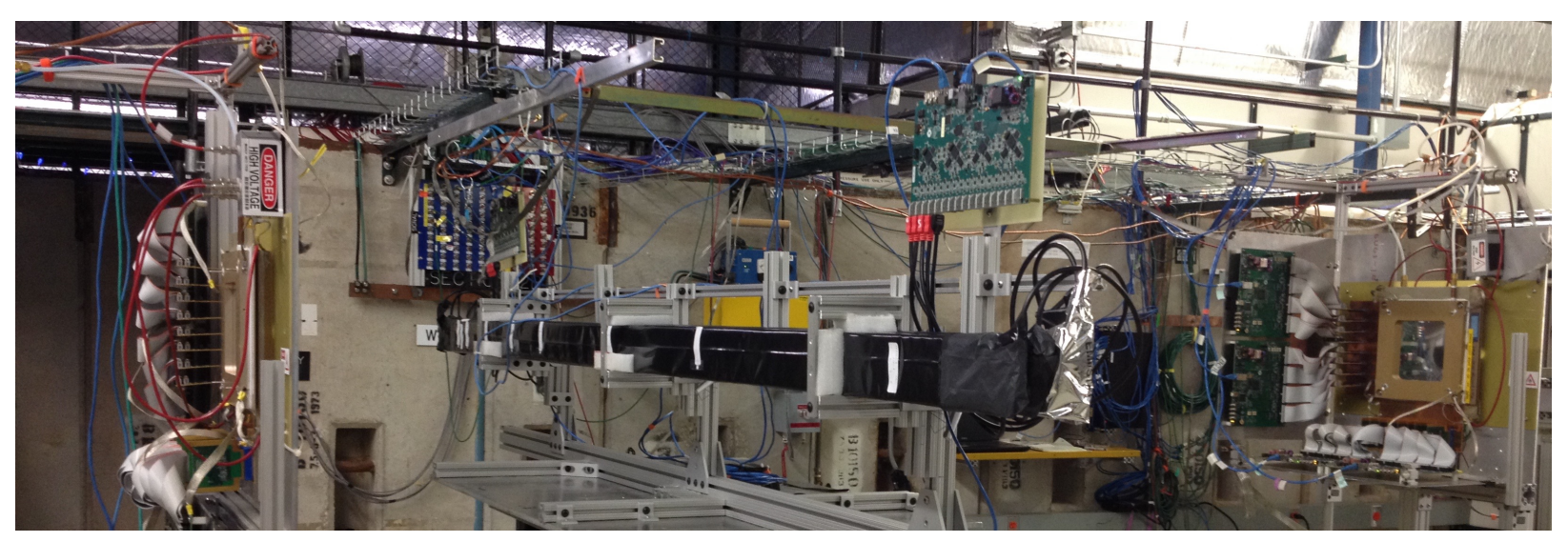

Figure 1. Photo of T-1043 setup showing CRV dicounters in their frame (center) mounted on the movable table.

\section{Setup:}

Dicounters were tested 4 at a time as shown in Figure 1. Front End Board (FEB) readout electronics were mounted above the dicounters and data was sent to the control room through ethernet connections. XY scans were done by remote operation of the movable table and the mounting frame could be manually tilted for angular scans.

\section{Results and Impact:}

Figure 2 shows the PE yield results for the best scintillator mix, taken in the June test beam period. The counter contained two $1.4 \mathrm{~mm}$ diameter fibers, each readout by a $2 \mathrm{~mm} \times 2$ $\mathrm{mm}$ SiPM. The red and blue curves are the signals in PE of each of the 2 SiPMs (means of 58 and 63 PE respectively) at one end of the counter, and the black curve is the sum of the readings (121 PE mean). For the $2 \mathrm{~cm}$ thick counters, the average $\mathrm{PE} / \mathrm{cm}$ for a single fiber 
was measured to be $\sim 30 \mathrm{PE} / \mathrm{cm}$, exceeding the requirement and confirming the baseline design. Publication of the full test beam results is pending.

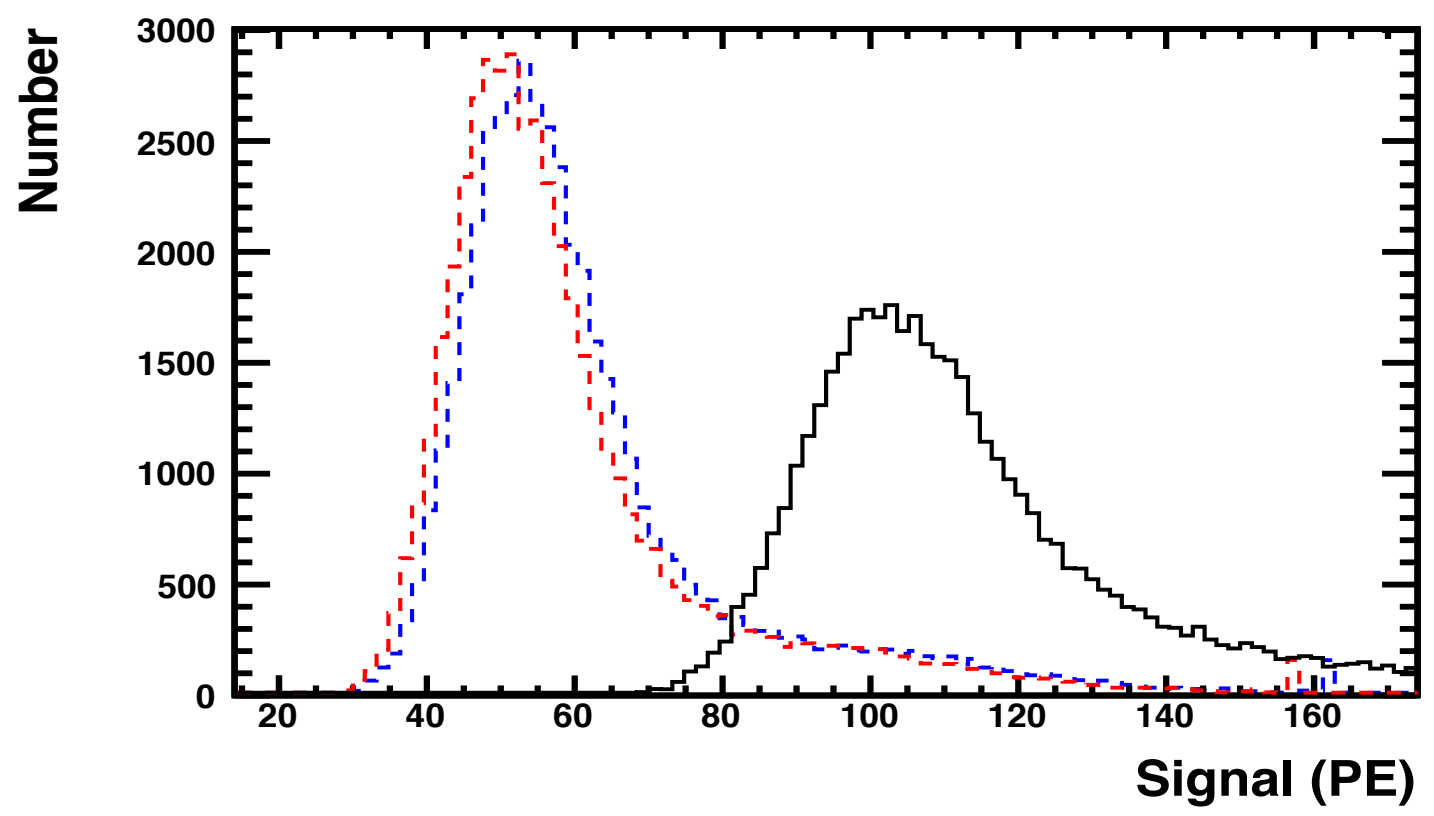

Figure 2. (Red,Blue) PE response for each of the 2 readout SiPMs, and (Black) PE sum for a counter. 
T-1044 / sPHENIX Calorimetry Test (V. Baily, R. Belmont, C. Biggs, J. Blackburn, S. Boose, C-Y. Chi, M. Chui, M. Connors, E. Desmond, A. Franz, J. Haggerty, X. He, M. Higdon, J. Huang, K. Kauder, E. Kistinev, J. Labounty, M. Lenz, W. Lenz, J. Lojoie, S. Li, D. Lynch, V. Loggins, T. Majoros, E.J. Mannel, E. O’Brien, M. Phipps, R. Pisani, C. Pinkenburg, S. Polizzo, C. Pontieri, M. Purschke, M. Sarsour, T. Rinn, R. Ruggiero, A. Sen, A. Sickles, M. Skoby, P. Sobel, S. Stoll, E. Thorsland, F. Toldo, B. Ujvari, S. Vazquez-Carson, C. Wood)

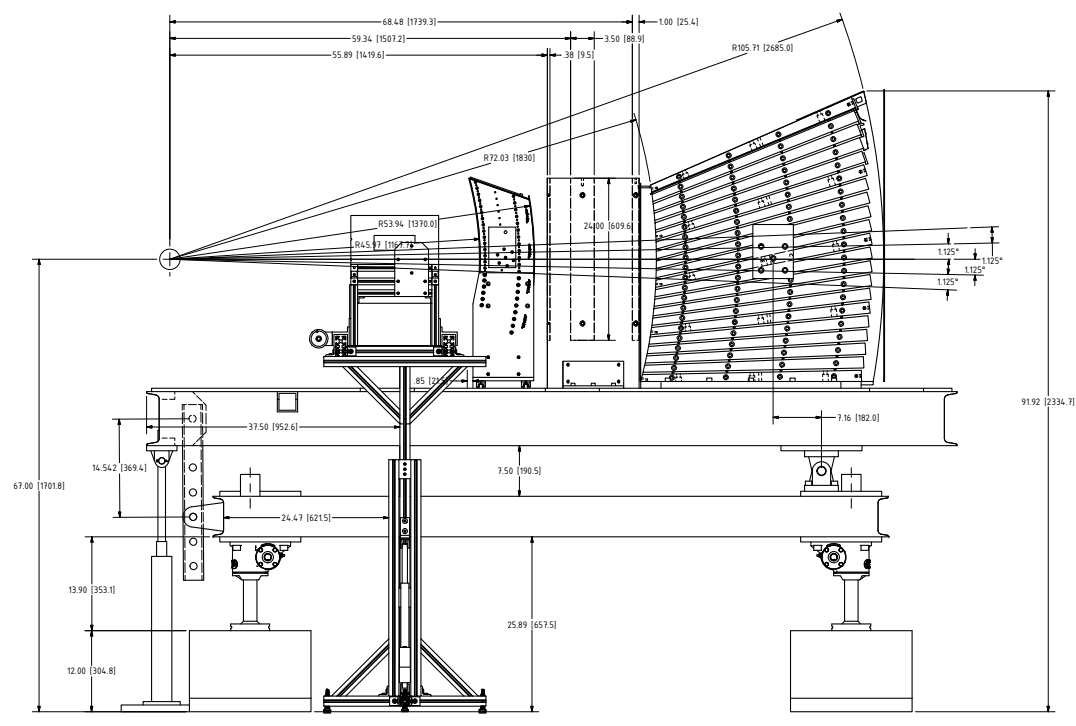

HORIZONTAL POSITION

Figure 1: Mechanical drawing showing the detectors in their nominal position centered on the beam line. From left to right are the EMCal, Inner HCal, Cryostat Mockup, Outer HCal. The beam is incident from the left.

Beam used: $1-60 \mathrm{GeV}$ Mixed beam, positive and negative polarity $120 \mathrm{GeV}$ Proton beam

Run dates: April 6 to May 3, 2016

\section{Motivation and Goals:}

The sPHENIX upgrade to the PHENIX detector is designed to study jets in heavy ion collisions at the Relativistic Heavy Ion Collider (RHIC) at Brookhaven National Laboratory and address questions about the nature of the perfect fluid, the quark-gluon plasma. The upgrade consists of the 1.5 Tesla BaBar superconducting solenoid, central tracking, electromagnetic and hadronic calorimeters with uniform coverage over a rapidity range of $|h|<1.1$ and $2 p$ in azimuth. The sPHENIX detector concept takes advantage of technological developments to enable a compact design with excellent performance. A tungsten-scintillator 
electromagnetic calorimeter read out with silicon photomultipliers (SiPMs) allows for a physically thin device, which can operate in a magnetic field, without the bulk of photomultiplier tubes and the need for high voltage distribution. The smaller electromagnetic calorimeter also allows the hadron calorimeter to be less massive, and the use of silicon photomultipliers for the hadron calorimeter allow for nearly identical electronic readout for the calorimeter systems.

Since the first run of T-1044 in 2014, the prototype calorimeters have been modified to reflect the new reference detector design and implement changes based on what was learned in the first run of T-1044. The design for the EMCal detector is a 1-D SPACAL design similar to the EMCal being designed at UCLA and tested in T-1018. The HCal detector has been modified to consist of 2 sections, inner and outer, with $1 \mathrm{l} 0$ of material separating the two sections to simulate the cryostat. The optical sensors used in the initial test beam run have been replaced with $40 \mathrm{~K} 15 \mathrm{~mm}^{2}$ micro-pixel devices. The increase in the number of pixels increases the dynamic range of the device, while the smaller feature sizes reduces the sensitivity of the devices to the effects of neutron radiation. While neutron radiation is not an issue for test beam operations it will be an issue for sPHENIX running at RHIC. The frontend amplifiers have been upgraded to improve the signal-to-noise and a switchable gain setting allows the detector to be calibrated on MIP signals from cosmic muons and primary protons.

The goals for this test beam experiment are to verify the performance of the electromagnetic and hadronic calorimeters for hadrons with energies ranging from a few $\mathrm{GeV}$ to $50 \mathrm{GeV}$ and a variety of geometrical orientations of the detectors to the impinging particles. In addition, the tests will allow testing of the next generation electronics for the calorimeter readout.

\section{Setup:}

The detector consisted of an EMCal module (64 towers in an 8 x 8 array), an inner HCal module (16 towers in a $4 \times 4$ array) and an outer HCal (16 towers in a $4 \times 4$ array) and is shown in Figure 1. Initially the EMCal was located on the motion table in the MT6.2C area allowing for detailed scans of the EMCal in a variety of orientations and different beam energies in order to understand the performance of the EMCal. The inner and outer HCal detectors were support structure in the MT6.2D area. The support structure allowed the entire $\mathrm{HCal}$ detector to be rotated $+/-5^{0}$ with respect to the beam line with a vertical adjustment to keep the detector centered on the beam line when rotated. In order to do combined measurements during the second half of the running, the EMCal was repositioned on a track directly in front of the Inner HCal. The track allowed the EMCal to be translated both horizontally and vertically with respect to the beam line independent of the HCal. 
The analog signals from both the EMCal and HCal were readout using a digitizer originally designed for the PHENIX Hadron Blind Detector (HBD) ${ }^{2}$. All channels were digitized with a 12-bit ADC operating at $60 \mathrm{MHz}$. Upon receipt of a beam trigger, 16 time samples for all channels were recorded for subsequent analysis. The system is capable of recording data at rates up to $7 \mathrm{kHz}$, although event rates at the MTEST facility were typically only a few $\mathrm{kHz}$ depending on the beam tune.

In addition to the calorimeters, the PMT signals from the 2 Cerenkov detectors were digitized and recorded for each trigger event. The gas pressures for each detector were adjusted based on the beam tune requested. In addition, a hodoscope consisting of $5 \mathrm{~mm}$ finger counters ( 8 in the vertical orientation and 8 in the horizontal orientation) was located immediately upstream of the EMCal. The signals from the $16 \mathrm{SiPMs}$ were digitized and recorded for each event.

A minbias using the MTest beam line scintillating counters, SC1, SC2, and SC3 in coincidence with the spill was used to trigger the DAQ. This allowed for a large sample of minimally biased events to be recorded for analysis each spill.

Data was collected over a 5 week period from April 6, 2016 to May 3, 2016 for a mixed beams ranging from 1 to $66 \mathrm{GeV}$ (both polarities) and $120 \mathrm{GeV}$ proton beams, with different positions and orientations of the detectors. In addition routine LED calibration data was collected for both calorimeters and cosmic ray data was collected for the HCal detector.

\section{Results and Impact:}

The primary focus of the on goinig data analysis is to understand the performance and energy resolution of the detectors. Studies include the calibration of the detectors both indivdually and as a combined system, response of the detector for different beam energies and particle composition, and enviromental variations such as temperature fluctuations. Figure 2 shows the preliminary energy resolution and linearity for the EMCal based on a preliminary MIP calibration. Electrons were selected using the beam line Cerenkov counters. A beam momentum spread of $\sim 2 \%$ is not unfolded ${ }^{3}$. Figure 3 shows a preliminary energy resolution spectrum for the combined EMCal and HCal detectors based on a calibration using cosmic rays on the left and on the right the linearity of the combined detector response over a range of $4-30 \mathrm{GeV}^{3}$. A preliminary 2 component fit gives a combined detector energy resolution of $\sim 70-80 \%+/-\sim 15 \%$ which meets the design requirement for the sPHENIX calorimeter.

\footnotetext{
${ }^{2}$ W. Anderson et al. Design, Construction, Operation and Performance of the Hadron Blind Detector for the PHENIX Experiment. Nucl. Instrum. Meth., A646:25, 2011

${ }^{3}$ C. Woody. Status and New Results for the sPHENIX Calorimeter Systems, CALOR 2016, Daequ Korea.
} 

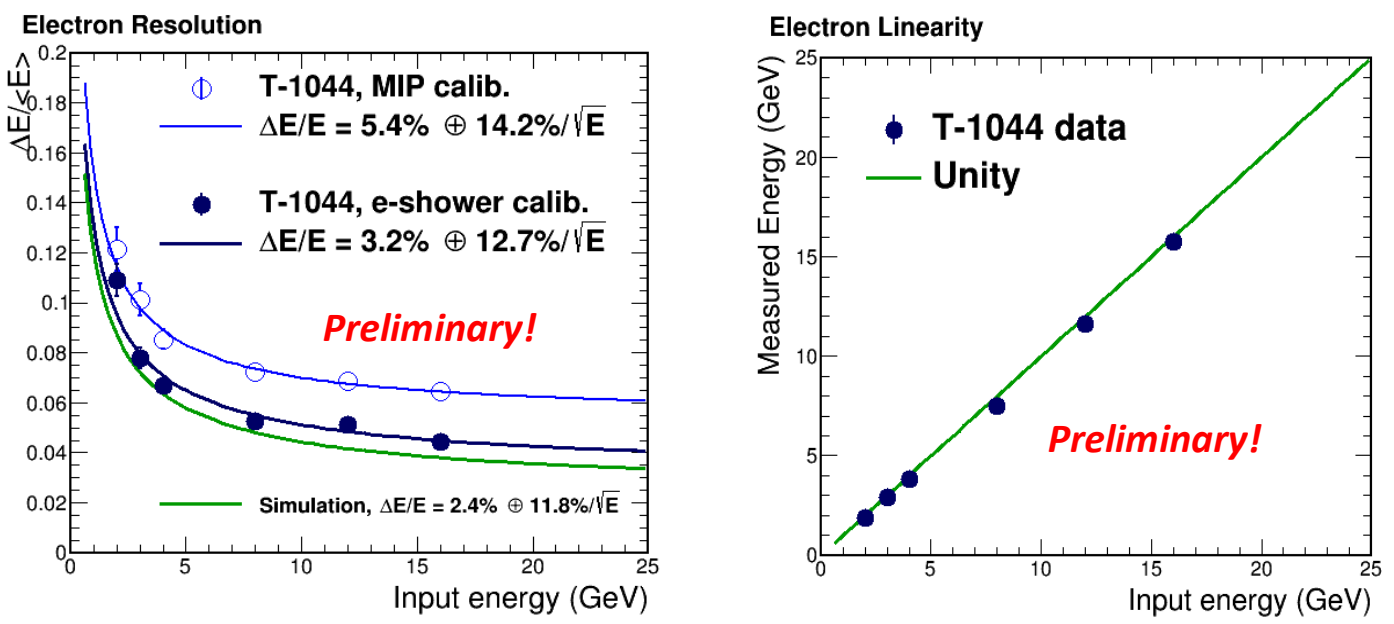

Figure 2: Preliminary EMCal energy resolution and linearity. A beam momentum spread of $\sim 2 \%$ is not unfolded. ${ }^{3}$
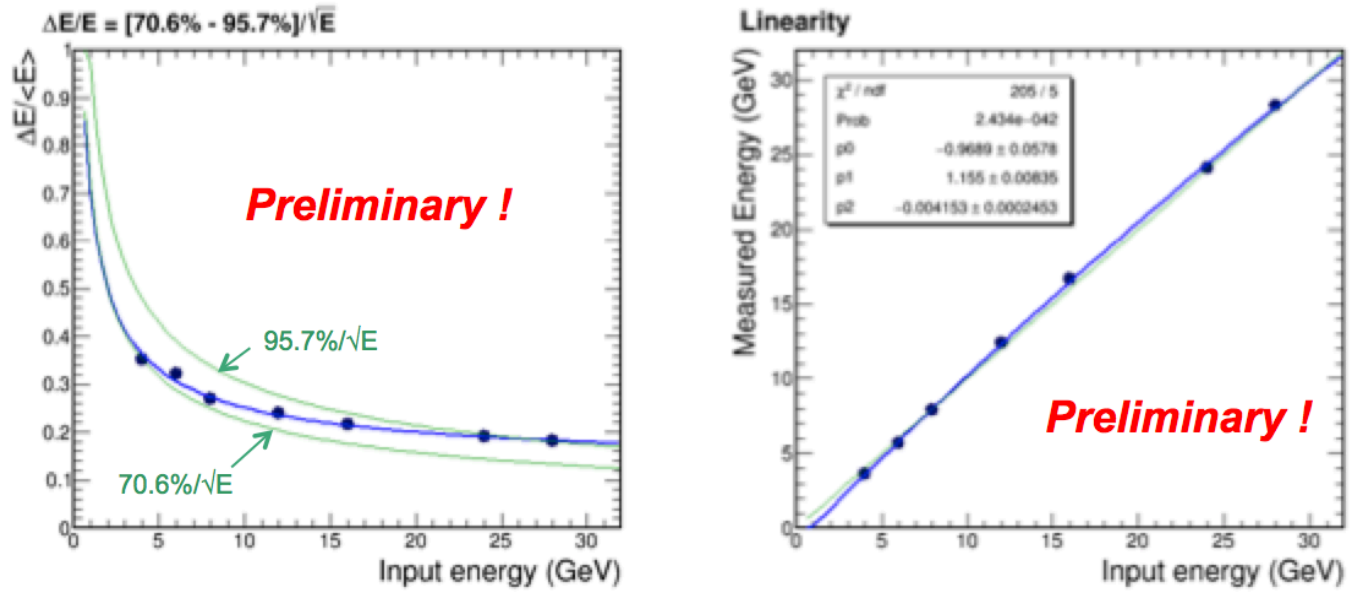

Figure 3: Preliminary energy resolution spectrums for the SPHENIX EMCal and HCal protoype calorimeters based on a cosmic ray calibration is shown on the left. On the right is the response linearity over the range of 4 to $28 \mathrm{GeV}^{3}$

Preliminary results of the April 2016 running of T-1044 were presented at CALOR 2016 and ICHEP 2016, with updated results to be presented in an invitred talk at the IEEE NSSMIC 2016 meeting. A manuscript for publication is also being prepared for submission in late 2016. 
T-1048 / EIC psTOF (M. Chiu, M. Alfred, M. Contalbrigo, X. He, A. Sukhanov, S. Syed, C.P. Wong ${ }^{4}$, J. Xie ${ }^{5}$ )

Beam used: $120 \mathrm{GeV}$ protons $4.8 \mathrm{GeV}$ pions

Run dates: $\quad 4 / 6 / 2016-5 / 3 / 2016$

Motivation and Goals:

The highest priority for a new facility the U.S. Nuclear Physics community is electron-Ion Collider (EIC), capable of colliding electrons on Ions or polarized protons at $1 / 3$ the center of mass energies of Hera, but with luminosities 100 times higher. As part of the eRD14 consortium,

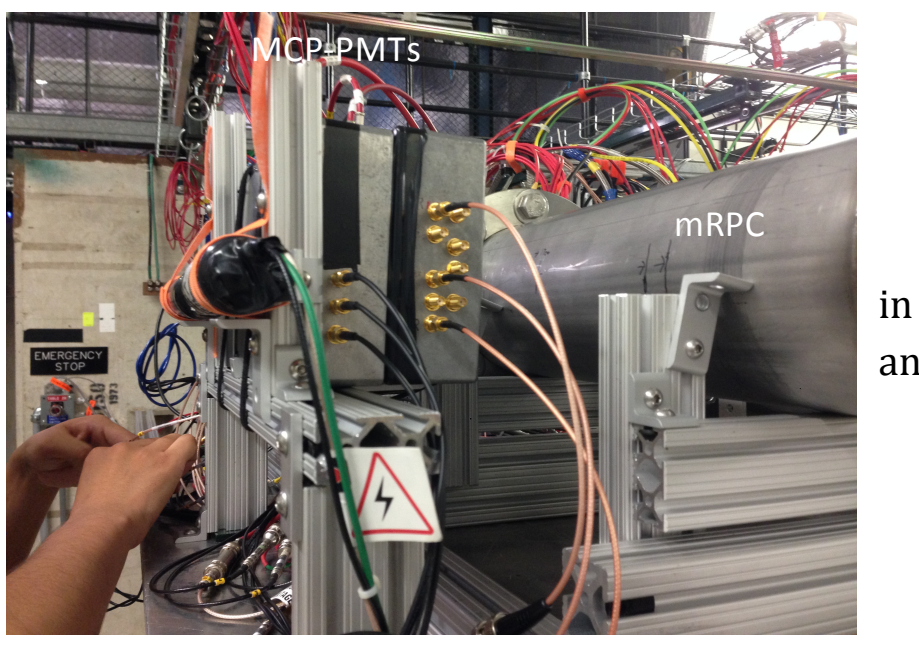

Fig. 1: MCP-PMTs in light tight boxes, with mRPCs behind it. Not shown is the AGEL mRICH. the authors are developing particle identification (PID) detectors to be used at the EIC. Three different technologies were tested during this run: $6 \times 6 \mathrm{~cm}^{2}$ LAPPD-style micro-channel plate PMTs (MCP-PMTs) from Argonne, a modular Aerogel RICH, and various multi-gap Resistive Plate Chambers (mRPCs).

\section{Setup:}

For the 3 different types of detectors under test, we aligned them one after another along the beamline. Due to the scattering from material we tested each detector separately, with the upstream detectors temporarily removed when needed. Most of the beam taken was with $120 \mathrm{GeV}$ protons, which provided the highest fluxes for testing the rate capability of the MCP-PMTs, and the cleanest most well understood beams for the mRPC and AGEL mRICH.

\section{Results and Impact:}

For the mRICH, we demonstrated that the Fresnel Lens indeed provides compact focusing of the Cerenkov ring onto a plane that is only $7.62 \mathrm{~cm}$ away. For the MCP-PMT, we demonstrated that the LAPPD style design rates as high as $100 \mathrm{kHz} / \mathrm{cm} 2$ can be sustained with no degradation in performance. We also demonstrated an initial timing resolution of at least 35 ps. For the mRPCs, which are usually made of glass, we attained good signals from the first ever 3D printed $\mathrm{mRPC}$. 
FERMILAB-TM- 2640-DI

November 2016

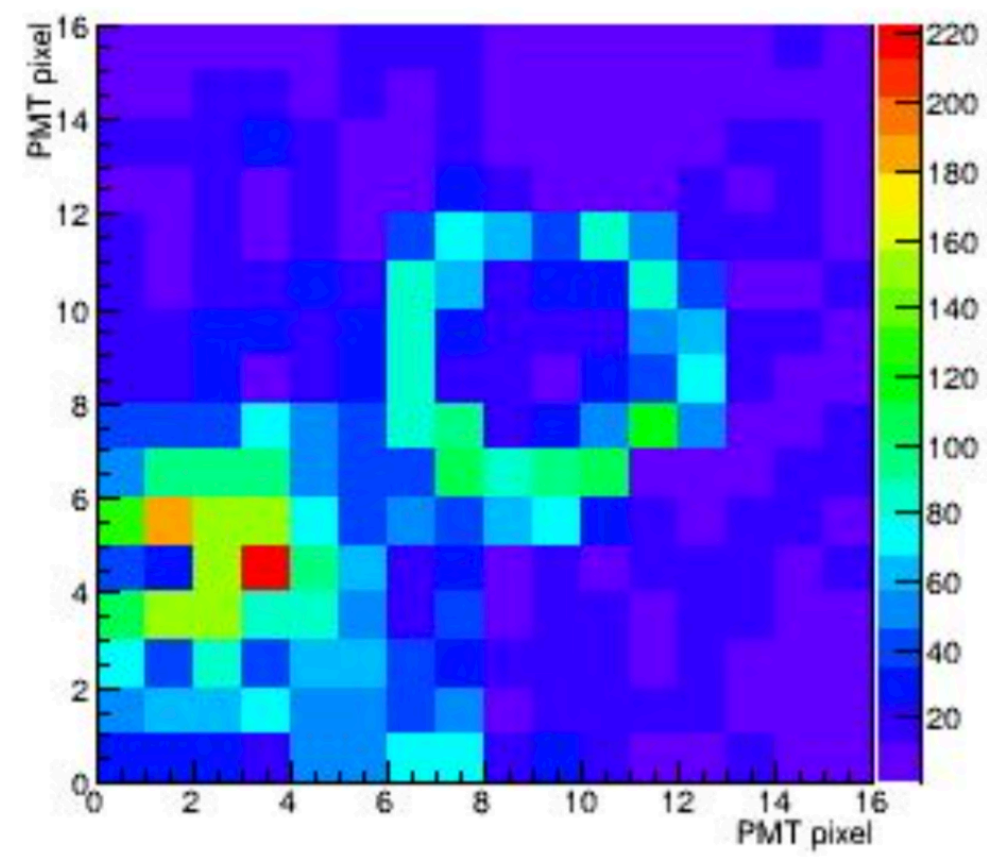

Figure 2: Cerenkov ring from AGEL mRICH, with particle incident on the lower left. 
T-1065 / Secondary Emitters Study (A. Ronzhin, G. Bolla, S. Los, E. Ramberg, A. Apresyan, S. Xie, M. Spiropulu, H. Kim).

Beam used: $120 \mathrm{GeV}$ protons, $4-32 \mathrm{GeV}$ electrons.

Run dates: $\quad$ March 2015, Dec 2015, June 2016.

\section{Motivation and Goals:}

The goal was to measure timing parameters of SiPMs, MCP, MCP- PMTs and Silicon for detecting MIPs and active layer of shower maximum (SM) detectors.
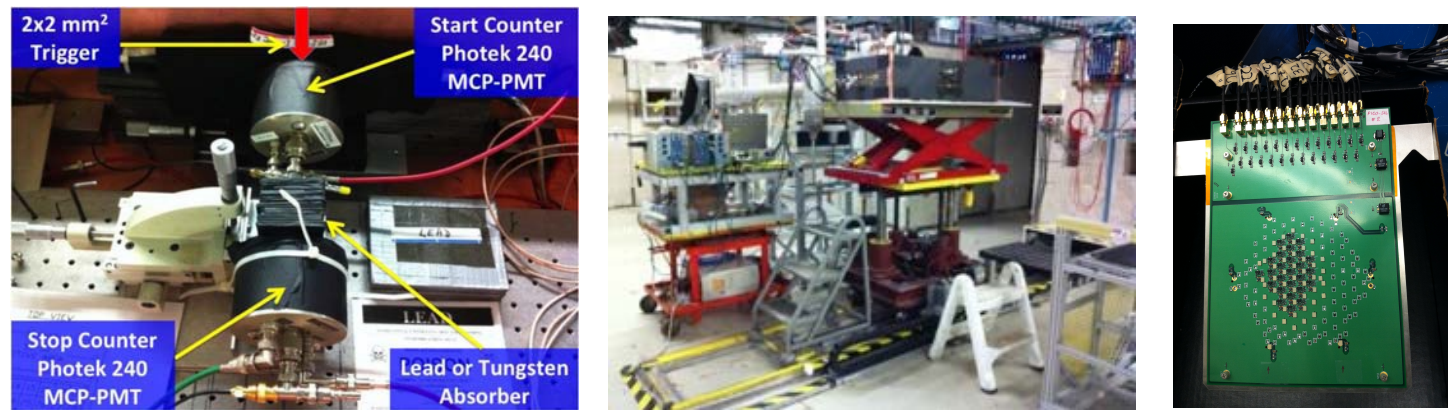

\section{Setup:}

The experiment was located in section $6.2 \mathrm{~b}$.

\section{Results and Impact:}

For SM based on MCP-PMT we obtained 13 ps time resolution and $\sim 11$ ps with sensitive layer based on silicon. We got 8.3ps time resolution for TOF, best in the world results.
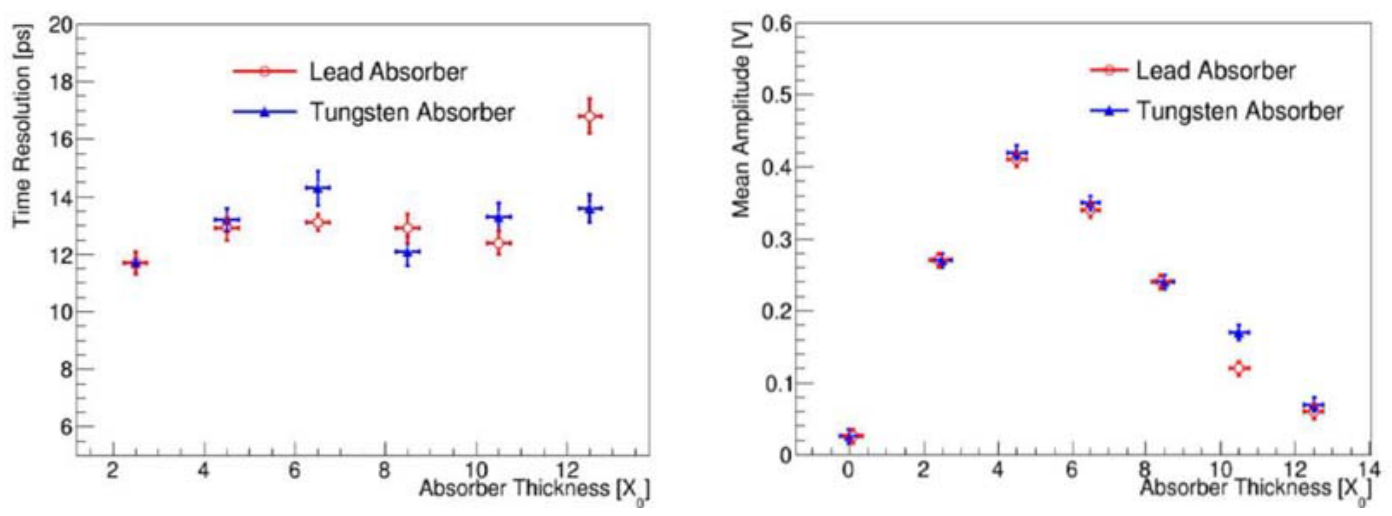
T-1068 / The Beam Test for the SVX4 Telescope (K.Hanagaki, Y.Ikegami, K.Yaiima, et al.)

Beam used: $120 \mathrm{GeV}$ proton

Run dates: $12 / 3-9 / 2016$

\section{Motivation and Goals:}

The purpose of this experiment is to study the silicon strip detector consisting of the silicon strip sensors and SVX4 readout ASIC's, called SVX4 telescope. This detector is used as the reference detector to measure the charged particle trajectory so that it provides the particle incident position at the other detector under test (DUT).

\section{Setup:}

We had four sets of the SVX4 telescopes that are sandwiched by two scintillators, one on upstream and the other on downstream. The coincidence of signals from the two scintillators is used as the trigger of the DAQ. In order to identify the particle incident position on the device under test, i.e. the single telescope, a track is reconstructed from hit positions of the remaining three telescopes. The signal from

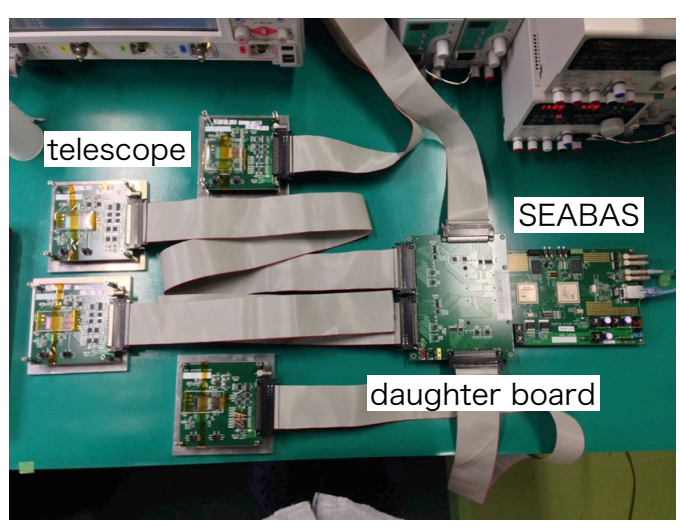
the telescopes are read out through the FPGA based DAQ board, so-called SEABAS, that transmits the signal to PC.

\section{Results and Impact:}

Right figure shows the residual distribution of one of the telescopes where two hits are found. In this case, the charge created by the incident particle is shared between the two strips, resulting in better resolution than in the case of one strip hit. The sigma of the narrow peak is about $4 \mathrm{um}$. Assuming the tracking resolution and the telescope's intrinsic resolution are the same, the position resolution is deduced to be roughly 3 um (=4/sqrt(2)). We also

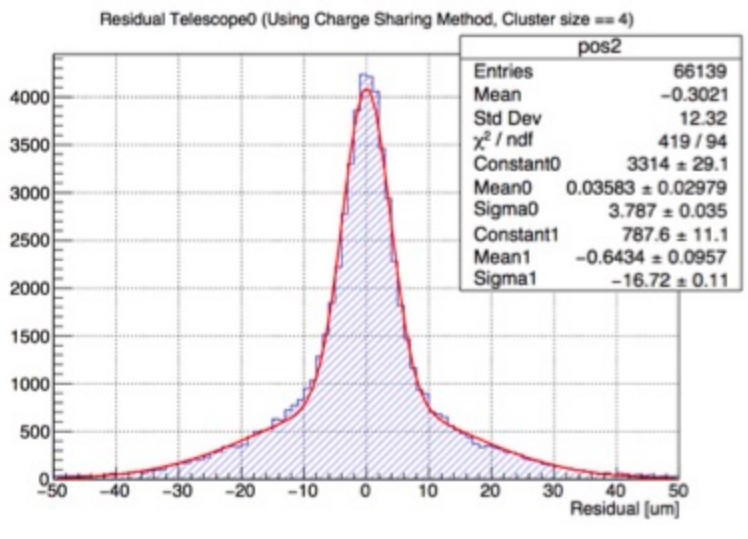
measured the efficiency of each telescope, and confirmed that all of them has the efficiency higher than $98 \%$. 
$\underline{\text { T-1072 / Muon strips (D. Denisov, V. Evdokimov, S. Lukić, P. Ujić) }}$

Beam used: $28 \mathrm{GeV}$ pion

Run dates: May $25^{\text {th }}-$ June $17^{\text {th }} 2016$

\section{Motivation and Goals:}

Beam test for muon strips is an experiment to measure the light yield, time resolution and longitudinal position resolution of scintillator strips for a muon system at future colliders.

The muon system at future colliders is envisioned as several layers of positionsensitive detectors embedded in the iron flux-return yoke of the solenoidal magnet. The role of the muon system is primarily the identification of muons and track

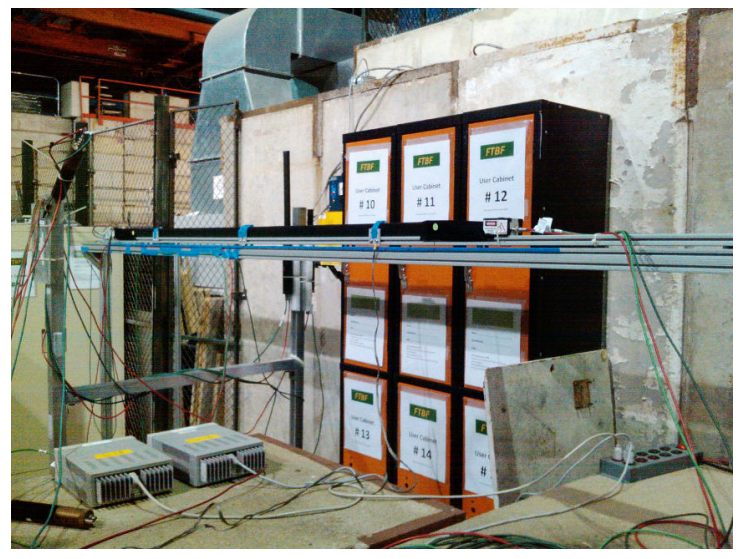

Figure 1: Photograph of the setup matching to the central tracker, besides serving as the tail catcher for the hadronic showers that penetrate beyond the hadron calorimetry. Previous tests performed at Fermilab with cosmic muons have shown that scintillators with wavelength-shifting (WLS) fibers and a SiPM readout offer promising performance (NIM A 823, 2016, pp.120-125). The purpose of the present beam tests is to determine the performance parameters with better accuracy and to measure the ultimate resolution achievable with present technologies.

\section{Setup:}

Tested strips were placed horizontally perpendicular to the beam on a sliding platform. Three trigger scintillators were arranged, two in front and one behind the tested strip, along the beam to signal muon passage in a narrow region $(2.5 \mathrm{~cm})$ around the beam center. The capability of the muon strip to measure the position of the muon impact depends on the measurement precision of the time difference of signals at both ends of the strip.

\section{Results and Impact:}

Time resolution of $320 \mathrm{ps}$ and position resolution of $\sim 5 \mathrm{~cm}$ were measured with the best of the tested strips. This result is record for this type of device, and fully adequate for the intended purpose. A publication with the results is in preparation for submission to NIM.

Prior to the beam test the muon flux has been measured at FTBF. The results have been described in the Fermilab technical memo FERMILAB-TM-2627-E. 
T-1073/ Beam Monitor for Mu2e (E. Prebys, D. Hedin, A. Gaponenko, R. Hooper, A. Dychkant, S. Uzunyan, D. Faia, R. Margraf, Z. Arcara, J. Waters)

\section{Beam Used:}

Run Period: June 29th-July $12^{\text {th }}, 2016$

\section{Summary of Tests:}

This run was to test the viability of the LDRD 2016.001 proposal for a Precision Time Profile Monitor (PTPM). ${ }^{4}$

Specifically, four quartz Cherenkov radiators were exposed to proton and other beams to measure their efficiency and accidental hit rates.

Reports

The run was summarized in Rachel Margraf's Lee Teng report ${ }^{5}$. To summarize, after optimizing thresholds:

- All four radiator/PMTs had efficiencies of $>99 \%$.

- The measured time resolution was $\mathrm{s}_{\mathrm{t}} \sim 1 \mathrm{~ns}$.

- The measured noise rate corresponds to an accidental four-fold coincidence within $10 \mathrm{~ns}$ of $<10^{-10}$.

These exceed the requirements of the PTPM.

${ }^{4}$ E. Prebys, D. Hedin, “Beam Precision Time Profile Monitor (PTPM)", BEAMS-DOC-5015 (2015)

5R. Margraf, "Beam Tests of Quartz Radiators for Precision Timing Profile Monitor", BEAMS-DOC-5221 (2016) 
T-1075 / Large-Area Fast Timing Beam Time of Flight (LAFTBToF) (J. Paley, Giulia Brunetti, Flavio Cavanna, Cheng-Ju Lin)

Beam used: LArIAT Tertiary Beam, cosmics

Run dates: $7 / 25 / 2016$ - present

\section{Motivation and Goals:}

Prototype large-area picosecond photodetectors (pLAPPDs), developed at Argonne National Laboratory (ANL), present an opportunity to provide identification of charged particles in the $2-10 \mathrm{GeV} / \mathrm{c}$ range. Traditional methods using ionization or Cherenkov detectors require large amounts of material or path-length for the particle to traverse in order to get a clean measurement, and are ill suited for particle beam identification in fixed-target experiments. The $6 \times 6 \mathrm{~cm}^{2}$ pLAPPD uses $\mathrm{mm}$-thick borosilicate glass micro-channel plates with a resistive surface coating added via atomic layer deposition. Electrons emitted by the

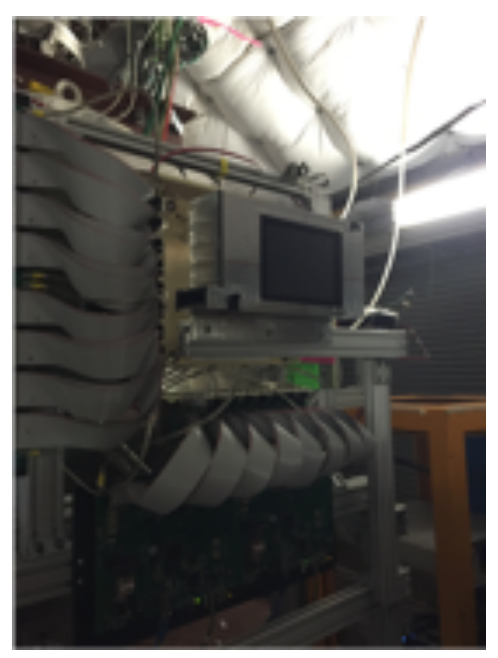
photocathode are accelerated through the MCPs, which have $\mu \mathrm{m}$-sized capillaries, and an avalanche of electrons is created as the electrons collide against the walls of the capillaries of the MCPs. The resulting electron avalanche is collected on anode striplines and the signal may be read out on both ends of the stripline. The resistive coating of the thin MCPs and the short path-length of the electrons to the readout results in fast rise times, gains of greater than $10^{6}$ and typical timing resolutions of 50 ps. Differential timing on the

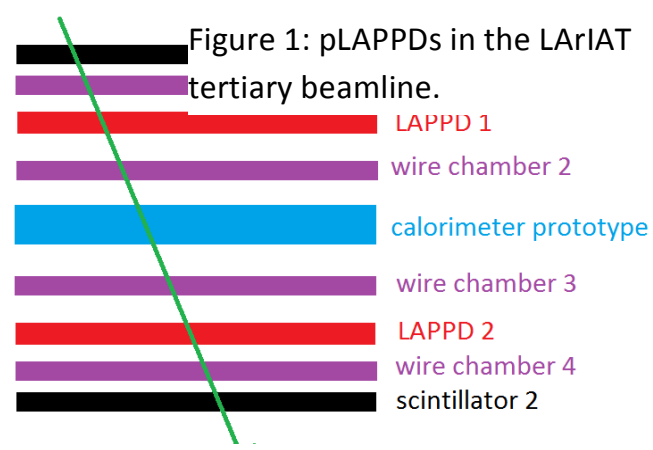

Figure 2: pLAPPDs in the cosmic ray telescope at the FTBF. ends of the striplines can provide mm-level position measurements. The material of the bare pLAPPD presents less than 0.05 of an interaction length and approximately 0.12 of a radiation length for a charged particle of $1 \mathrm{GeV} / \mathrm{c}$. In our application, two pLAPPDs separated by 5-10 meters can be used in a time-of-flight (ToF) measurement for $3 \sigma$ separation of 2-7 GeV/c charged pions, kaons and protons with minimal impact on the particle's trajectory and momentum.

The goal of this test-beam study is to measure the efficiency, timing resolution and position resolution the pLAPPDs using both FTBF beam, as shown in Fig. 1 and cosmic rays using the FTBF cosmic ray telescope, as depicted in Fig. 2. 


\section{Setup:}

A pLAPPD was installed in the LArIAT tertiary beamline as shown in the photo in Fig. 1, only a few days before the 2016 accelerator shutdown, and read out via the LArIAT DAQ. Unfortunately it discovered too late that we were looking at the incorrect electronics channel in the LArIAT data quality monitor, and that we were in fact getting no signals from the device. Upon investigation of the pLAPPD board, it was discovered that key components (a resistor and a capacitor) were missing from the board and there was no HV across the MCPs in the device. Around the time this was discovered, the beam turned off.

We are now working on testing the pLAPPDs by reading the single-pe noise signals in a digital oscilloscope (e.g., see Fig. 3). They will soon be mounted in the FTBF muon telescope as depicted in Fig. 2, and again read out by the LArIAT DAQ. The muon telescope setup will be used until beam returns.

\section{Results and Impact:}

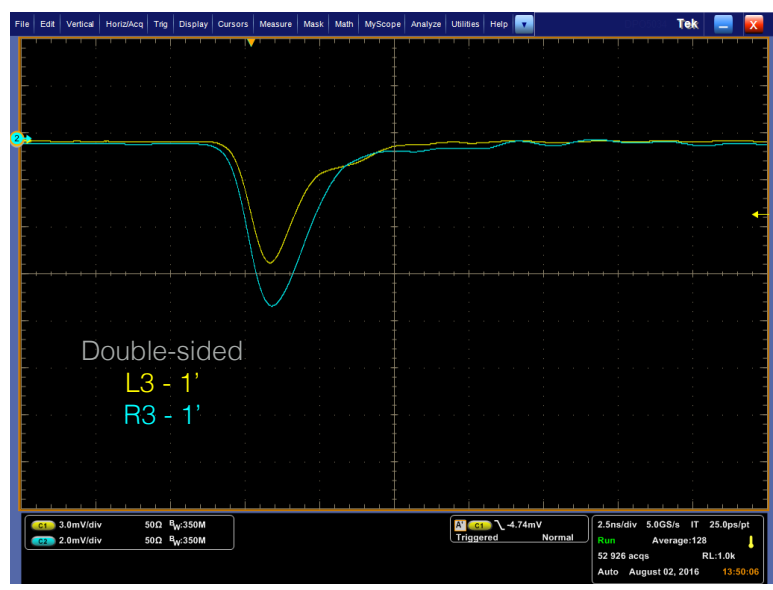

At this point we have no results to report.

Figure 3: Digital oscilloscope screen-capture of a single photoelectron noise signal readout on both ends of a stripline in a PLAPPD. 


\section{Future projects at the Test Beam}

The test beam facility is an important resource for Fermilab and the community. In order to continue to serve our users in the best possible way, we have a plan to continue to improve the quality of the facility. We have also taken a look at the possible users that could come over the next few years and have held several meetings to gather information from different groups interested in both MTest and in MCenter.

The next few years will see the end of E906/SeaQuest (FY17) and another long shutdown at CERN. Both of these events will impact our users at the test beam. We hope to maintain our current running, using $10 \%$ of the timeline as we do when SeaQuest is running. We can expect that our usage will increase during the Cern LS2 (long Shutdown 2), as it did during the first long shutdown of the test beams at CERN. In order to prepare for that, we will improve the facility on multiple fronts. First and foremost, we will enhance beamline instrumentation as suggested by the committee last meeting. We were able to start work on studies this past year, as shown in section 1.3, and made considerable progress. We will further our beam studies with a new Time of Flight system and spectrometer.

The areas we will work to improve on are:

- Characterize the beam in MTest and MCenter. This entails taking measurements at all energies with all the configurations possible (collimators, helium tubes, etc).

- Develop a DAQ that is easy to use for both users and facility studies.

- Develop analysis tools for users.

- Purchase/develop infrastructure for $\mathrm{HV}, \mathrm{ToF}$, and a spectrometer.

During the year, we will take advantage of opportunities to perform beam studies while beam available. When we are not able to use beam, we will use the time to analyze the data collected and work on analysis tools and simulations for users.

To summarize, our plan over the next few years is:

- Build a spectrometer and time of flight system (note these are both in progress)

- Develop analysis tools for users (this is also in progress, with much work done by D. Jensen)

- Characterize the beamline, starting with low energy particle composition followed by medium energy composition.

- Finish the unified data acquisition system.

- When adding new experiments to MCenter, use the improvements to benefit future users also.

- We will also continue to solicit feedback from users on improvements at the test beam. 


\section{Listing of Test Beam Publications}

1. Test Beam Studies of Silicon Timing for Use in Calorimetry.

A. Apresyan, G. Bolla, A. Bornheim, H. Kim, S. Los, C. Pena, E. Ramberg,

A. Ronzhin, M. Spiropulu, and S. Xie. NIM, A825 (2016) 62-68.

2. High time resolution photo-detectors for PET applications, Anatoly Ronzhin, invited by NIM editor article, Special Medical Edition. NIM, A809 (2016), 53-57.

3. Direct tests of micro channel plates as the active element of a new shower maximum detector,

A. Ronzhin, S. Los, E. Ramberg, A. Apresyan, S. Xie, M. Spiropulu, H.

Kim. NIM, A795 (2015), 52-57.

4. Study of the timing performance of micro channel plate photomultiplier for use as an active layer in a shower maximum detector.

A. Ronzhin, S. Los, E. Ramberg, A. Apresyan, S. Xie, M. Spiropulu, H.

Kim, NIM, A795 (2015), 288-292.

5. A feasibility study of a PET/MRI insert detector using strip-line and waveform sampling data acquisition, H. Kim, C.-T. Chen, N. Eclov, A. Ronzhin, P. Murat, E. Ramberg, S. Los, Alice M Wyrwicz, Limin Li, C.-M. Kao, NIM, A784 (2015), 557-564.

6. On Timing Properties of LYSO-based Calorimeters, D. Anderson, A. Apresyan, A. Bornheim, J. Duarte, C. Pena, A.

Ronzhin, M. Spiropulu, J. Trevor, S. Xie, NIM, A794 (2015) 7-14.

7. Precision timing measurements for high energy photons, Dustin Anderson, Artur Apreysan, Adi Bornheim, Javier Duarte, Harvey Newman, Cristian Pena, Anatoly Ronzhin, Maria Spiropulu, Jason Trevor, Si Xie, Ren-Yuan Zhu, NIM, A787 (2015) 94-97.

8. Development of a new fast shower maximum detector based on microchannel plate photomultipliers (MCP-PMT) as an active elements, A. Ronzhin, S. Los, E. Ramberg, A. Apresyan, S. Xie, M. Spiropulu, H. Kim, A. Zatserklyaniy, NIM, A759 (2014), 63-73.

9. Status of ADRIANO R\&D in T1015 Collaboration, C. Gatto et al. 2015 J. Phys.: Conf. Ser. 587012060 .

10. Preliminary Results from a Test Beam of ADRIANO Prototype, C. Gatto et al. 2012 J. Phys.: Conf. Ser. 404012030 